Homology, Homotopy and Applications, vol.11(1), 2009, pp.315-348

\title{
STEENROD OPERATIONS ON THE NEGATIVE CYCLIC HOMOLOGY OF THE SHC-COCHAIN ALGEBRAS
}

\author{
CALVIN TCHEKA
}

(communicated by Charles Weibel)

\begin{abstract}
In this paper we prove that the Steenrod operations act naturally on the negative cyclic homology of a differential graded algebra $A$ over the prime field $\mathbb{F}_{p}$ satisfying some extra conditions. When $A$ denotes the singular cochains with coefficients in $\mathbb{F}_{p}$ of a 1-connected space $X$, these extra conditions are satisfied. The Jones isomorphism identifies these Steenrod operations with the usual ones on the $S^{1}$-equivariant cohomology of the free loop space on $X$ with coefficients in $\mathbb{F}_{p}$. We conclude by performing some calculations on the negative cyclic homology.
\end{abstract}

\section{Introduction}

Since their construction by N. Steenrod [22], Steenrod operations have played a central role in homotopy theory and in representation theory. In the topological setting, Steenrod operations $\left\{P^{i}\right\}_{i \in \mathbb{N}}$ are stable natural transformations

$$
P^{i}: H^{*}\left(-; \mathbb{F}_{p}\right) \rightarrow \begin{cases}H^{*+i}\left(-; \mathbb{F}_{p}\right) & \text { if } p=2 \\ H^{*+(p-1) i}\left(-; \mathbb{F}_{p}\right) & \text { if } p \text { is an odd prime }\end{cases}
$$

where $H^{*}\left(-; \mathbb{F}_{p}\right)$ denotes the singular cohomology functor with coefficients in the prime field $\mathbb{F}_{p}$. When $p=2, P^{i}$ is called an $i$-Steenrod square and usually denoted by $S q^{i}$, while when $p$ is an odd prime, $P^{i}$ is called an $i$-Steenrod power. These transformations satisfy the following properties:

1. $P^{0}=i d$.

2. $P_{\left.\right|_{H^{k}\left(-, \mathbb{F}_{p}\right)} ^{i}}^{i}=0,($ resp. $\xi)$ if $\begin{cases}i>k & (\text { resp. } i=k) p=2 \\ i>2 k & \text { (resp. } i=2 k) p \text { is an odd prime. }\end{cases}$

3. $P^{k}(-\cup-)=\sum_{i+j=k} P^{i}-\cup P^{j}-$, (the Cartan formula).

4. The Adem relations (see [16], pages 129 and 367).

Here $i d$ (respectively 0) denotes the identity transformation (respectively the constant transformation whose value is 0 ) while $\xi$ denotes the Frobenius transformation $x \mapsto$

Received February 22, 2008, revised December 16, 2008; published on June 12, 2009.

2000 Mathematics Subject Classification: 55S20, 57T30, 54C35, $13 \mathrm{D} 03$.

Key words and phrases: Hochschild homology, negative cyclic homology, bar and cobar construction, shc-algebra and $\pi$-shc-algebra.

This article is available at http://intlpress.com/HHA/v11/n1/a13

Copyright (c) 2009, International Press. Permission to copy for private use granted. 
$x^{p}$. A. Dold [4] defined them in a more general context replacing the singular chains on a topological space by an arbitrary simplicial coalgebra. Later P. May [15] gave a purely algebraic construction of Steenrod operations which leads to the notion of $E_{\infty}$-algebra, as recently developed by Mandell [14] in homotopy theory, and to the construction of Steenrod operations in other settings. For example, there exist Steenrod operations on

- the cohomology of a commutative $\mathbb{F}_{p}$-Hopf algebra due to A. Liulevicius [12], after the paper of Dold quoted above;

- the cohomology of a restricted $p$-Lie algebra due to P. May [15];

- the cohomology of non commutative $p$-differential forms due to M. Karoubi [11];

- the cyclic cohomology of a commutative $\mathbb{F}_{p}$-Hopf algebra due to M. Elhamdadi and Y. Gouda [5].

Let us recall here that if $\left\{V^{i}\right\}_{i \in \mathbb{N}}$ is a graded $\mathbb{F}_{p}$-vector space and if $T^{c}(s V)$ denotes the free coalgebra generated by the suspension of $V$, denoted $s V$, then:

- $V$ is an $A_{\infty}$-algebra if there exists a degree 1 coderivation $D$ on $T^{c}(s V)$ such that $D \circ D=0$ and $D_{\left.\right|_{T^{0}(s V)}}=0$.

- $V$ is a $B_{\infty}$-algebra if it is an $A_{\infty}$-algebra and if there exists a product on $T^{c}(\mathrm{~s} V)$ such that $T^{c}(\mathrm{sV})$ is a differential graded Hopf algebra.

- $V$ is a $C_{\infty}$-algebra if it is an $A_{\infty}$-algebra such that $T^{c}(s V)$ is a differential graded Hopf algebra for the shuffle product.

While it is possible to define the Hochschild homology (and the negative cyclic homology) of an $A_{\infty}$-algebra $[\mathbf{9}, \mathbf{8}]$, the lack of associativity of these operadic algebras complicates explicit computations. Hopefully, strongly homotopy commutative algebras (shc for short), as introduced by H.J. Munkholm [17], will considerably simplify the above mentioned calculations. They are associative $B_{\infty}$-algebras. Moreover, it is known that:

- The normalized singular cochain complex with coefficients in $\mathbb{F}_{p}$ of a connected space $X, C^{*}\left(X ; \mathbb{F}_{p}\right)$, is a shc-algebra [17].

- The Hochschild homology of a shc-algebra $A$ with coefficients in $A, H H_{*}(A ; A)$, is a graded algebra [20].

- The negative cyclic homology of a $s h c$-algebra $A, H C_{*}^{-}(A)$, is a graded algebra $[18]$.

- Let $X$ be a 1-connected space and $L X$ be the free loop space. That is, $L X=$ $\operatorname{Map}\left(S^{1}, X\right)$ is the space of continuous maps from $S^{1}$ to X endowed with the compact open topology. The Jones isomorphism $H H_{*}\left(C^{*}\left(X, \mathbb{F}_{p}\right) ; C^{*}\left(X, \mathbb{F}_{p}\right)\right) \rightarrow$ $H^{*}\left(L X ; \mathbb{F}_{p}\right)$ is a homomorphism of graded algebras $[\mathbf{2 0}]$.

- Let $X$ be a 1-connected space and $L X$ the free loop space. The Jones isomorphism $H C_{*}^{-}\left(C^{*}\left(X, \mathbb{F}_{p}\right)\right) \rightarrow H_{S^{1}}^{*}\left(L X ; \mathbb{F}_{p}\right)$ is a homomorphism of graded algebras $[\mathbf{1 8}]$.

Here $H_{S^{1}}^{*}\left(L X ; \mathbb{F}_{p}\right)$ denotes the $S^{1}$-equivariant cohomology of $L X$ with coefficients in $\mathbb{F}_{p}$.

B. Ndombol and Jean-Claude Thomas [21] have introduced the notion of $\pi$-shcalgebra and have proved that 
- The normalized singular cochain complex with coefficients in $\mathbb{F}_{p}$ of a connected space $X, C^{*}\left(X ; \mathbb{F}_{p}\right)$, is a $\pi$-shc-algebra $[\mathbf{2 1}]$.

- There exist Steenrod operations on the Hochschild homology of a $\pi$-shc-algebra $A$ with coefficients in $A$.

- Let $X$ be a 1-connected space and $L X$ be the free loop space.

The Jones isomorphism $H H_{*}\left(C^{*}\left(X, \mathbb{F}_{p}\right) ; C^{*}\left(X, \mathbb{F}_{p}\right)\right) \rightarrow H^{*}\left(L X ; \mathbb{F}_{p}\right)$ respects the Steenrod operations.

In this paper we complete the above result in proving:

Theorem 1.1. Let $\left(\left(A, d_{A}\right), \mu_{A}, \kappa_{A}\right)$ be a $\pi$-shc cochain algebra as in [21].

1. The negative cyclic homology of a differential graded $\pi$-shc algebra $A$ with coefficients in $A, H C_{*}^{-}(A)$, has algebraic Steenrod operations.

2. Let $X$ be a 1-connected space and $L X$ be the free loop space.

The Jones isomorphism $H C_{*}^{-}\left(C^{*}\left(X, \mathbb{F}_{p}\right)\right) \rightarrow H_{S^{1}}^{*}\left(L X ; \mathbb{F}_{p}\right)$ respects the Steenrod operations. (See $[\mathbf{1 8}, \mathbf{1 0}]$.)

The Steenrod operations, considered in our theorem, are defined at the chain level and satisfy the properties:

1. $P^{0}(1)=1$ if 1 denotes the unit of the graded algebra $H C_{*}^{-}(A)$.

2. $P_{\left.\right|_{H C_{k}^{-}\left(-, \mathbb{F}_{p}\right)} ^{i}}^{i}=0,($ resp. $\xi)$ if $\begin{cases}i>k & \text { (resp. } i=k) p=2 \\ i>2 k & \text { (resp. } i=2 k) p \text { is an odd prime. }\end{cases}$

3. $P^{k}(-\cup-)=\sum_{i+j=k} P^{i} \cup P^{j}$, the Cartan formula.

Except if $A=C^{*}\left(X ; \mathbb{F}_{p}\right)$, the Steenrod operations constructed by Ndombol-Thomas or those considered in part 1 of our theorem do not in general satisfy the Adem relations. An operadic construction as in [2] or [1] allows us to define an action of the large Steenrod algebra on the Hochschild homology of a $E_{\infty}$-algebra. Such an action on the negative cyclic homology remains an open question. In these notes, quasiisomorphism means a homomorphism which is an isomorphism in (co)homology.

The paper is organized as follows. Section 2 is a recollection of definitions. Part 1 (respectively Part 2) of Theorem 1.1 is proved in Section 3 (respectively Section 4). Recalling the $\pi$-shc-minimal model and explicit computations are the subjects of Section 5 and Section 6 respectively. This paper is a part of my thesis supervised by professors B. Ndombol and J. C. Thomas of Yaounde I University, Cameroon, and Angers University, France respectively.

\section{Convention}

Throughout this paper, we use the Kronecker convention: an object with lower negative graduation has upper non-negative graduation.

\section{Preliminaries}

Let $\pi$ be any finite group and $p$ a fixed prime. Throughout this paper, we work over the field $\mathbb{F}_{p}$ equipped with the trivial action of $\pi$. The ring group $\mathbb{F}_{p}[\pi]$ is an augmented algebra. 


\subsection{Algebraic Steenrod operations}

The material involved here is contained in [17]. Let $\pi=\left\{1, \tau, \ldots, \tau^{p-1}\right\}$ be the cyclic group of order $p$. Let $W \stackrel{\varepsilon_{W}}{\longrightarrow} \mathbb{F}_{p}$ be a projective resolution of $\mathbb{F}_{p}$ over $\mathbb{F}_{p}[\pi]$; that is $W=\left(W_{i}\right)_{i \geqslant 0} ; W_{i} \stackrel{\delta_{i}}{\longrightarrow} W_{i-1} ; W_{0} \simeq \mathbb{F}_{p}[\pi]$, where each $W_{i}$ is a right projective $\mathbb{F}_{p}[\pi]$ module and $\delta_{i}$ is $\pi$-linear. We choose $\mathbb{F}_{p} \stackrel{\eta_{W}}{\longrightarrow} W$ such that $\varepsilon_{W} \circ \eta_{W}=i d_{\mathbb{F}_{p}}$. Necessarily $\eta_{W} \circ \varepsilon_{W} \simeq i d_{W}$.

Let $A=\{A\}_{i \in \mathbb{Z}}$ be a differential graded algebra (not necessarily associative). We denote by $m_{A}^{(p)}$ (resp. $\left.\left(H m_{A}\right)^{(p)}\right)$ the iterated product $a_{1} \otimes a_{2} \otimes \cdots \otimes a_{p} \mapsto a_{1}$. $\left(a_{2}\left(\cdots a_{p}\right)\right.$ ) (resp. the iterated product induced on $H A$ by $\left.m_{A}^{(p)}\right)$.

Identify $\tau \in \pi$ with the $p$-cycle $(p, 1, \ldots, p-1)$ and assume that $\pi$ acts trivially on $A$, thus $\pi$ acts diagonally on $A^{\otimes p}$ and on $W \otimes A^{\times p}$.

If the natural map $H\left(W \otimes A^{\otimes p}\right) \stackrel{\cong}{\longrightarrow} H(A)^{\otimes p}{\stackrel{\left(H m_{A}\right)}{\longrightarrow}}^{(p)} H A$ lifts to a $\pi$-linear chain map $\theta: W \otimes A^{\otimes p} \longrightarrow A$, then for any $i \in \mathbb{Z}$ and $x \in H^{n} A$, there exists a well defined class

$$
P^{i}(x) \in \begin{cases}H^{n+i}(A) & \text { if } p=2 \\ H^{n+2 i(p-1)}(A) & \text { if } p>2\end{cases}
$$

such that:

1.

$$
P^{i}\left(1_{H A}\right)=0 \text { if } i \neq 0 .
$$

2.

$$
\text { If } p=2, \begin{cases}P^{i}(x)=0 & \text { if } i>n \\ P^{i}(x)=x^{2} & \text { if } i=n\end{cases}
$$

3.

$$
\text { If } p>2, \begin{cases}P^{i}(x)=0 & \text { if } 2 i>n \\ P^{i}(x)=x^{p} & \text { if } n=2 i .\end{cases}
$$

Moreover these classes do not depend on the choice of the resolution $W$ nor $\eta$ and are compatible with algebra homomorphisms commuting with structural map $\theta$.

These operations do not in general satisfy $P^{i}(x)=0$ if $i<0, P^{0}(x)=x$, the Cartan formulas and the Adem relations.

\subsubsection{Cartan formula}

Let us consider the differential graded algebra $A=\left\{A^{i}\right\}_{i \in \mathbb{Z}}$ such that $A^{i}=0$ for $i<0$.

A homogeneous map $W \stackrel{f}{\longrightarrow} A$ has a degree $k$ if $f \in \operatorname{Hom}^{k}(W, A)=\prod_{i \geqslant 0} \operatorname{Hom}\left(W_{i}\right.$, $\left.A^{k-i}\right)=\bigoplus_{i=0}^{k} \operatorname{Hom}\left(W_{i}, A^{k-i}\right)$.

The differential of $f$ is $D(f)=d \circ f-(-1)^{|f|} f \circ \delta ; \pi$ acts on each $\operatorname{Hom}^{k}(W, A)$ by $(\sigma f)(w)=f(w \sigma)$; the evaluation map

$$
\begin{aligned}
\operatorname{Hom}(W, A) & \stackrel{e v_{0}}{\longmapsto} A \\
f & \longmapsto e v_{0}(f)=f\left(e_{0}\right)
\end{aligned}
$$

is a homomorphism of chain complexes. 
Let $W \stackrel{\psi_{W}}{\longrightarrow} W \otimes W$ be any diagonal approximation and denote by $m_{A}$ the product on the algebra $A$. We have the cup-product

$$
\begin{aligned}
\operatorname{Hom}^{k}(W, A) \otimes \operatorname{Hom}(W, A) & \stackrel{\cup}{\longrightarrow} \stackrel{k+l}{\operatorname{Hom}}(W, A) \\
f \otimes g & \longmapsto f \cup g=m_{A} \circ(f \otimes g) \circ \psi_{W}
\end{aligned}
$$

that defines a nonassociative differential graded algebra structure on $\operatorname{Hom}(W, A)$.

Proposition 2.1 ([21]). If $A=\left\{A^{i}\right\}_{i \in \mathbb{Z}}$ is differential graded algebra such that $A^{i}=$ 0 if $i<0$ and $\theta$ the structural map as in 2.1, then:

1. The structural map $W \otimes A^{\otimes p} \stackrel{\theta}{\longrightarrow} A$ induces a $\pi$-chain map

$$
\begin{aligned}
A^{\otimes p} & \stackrel{\tilde{\theta}}{\longrightarrow} \operatorname{Hom}(W, A) \\
u & \longmapsto \begin{cases}W & \stackrel{\tilde{\theta}(u)}{\longrightarrow} A \\
w & \longmapsto \tilde{\theta}(u)(w)=(-1)^{|u||w|} \theta(w \otimes u)\end{cases}
\end{aligned}
$$

such that $e v_{0} \circ \tilde{\theta}=m_{A}^{(p)}$ and $H\left(e v_{0}\right) \circ H(\tilde{\theta})=H\left(m_{A}\right)^{(p)}$.

2. If we assume that $H(\tilde{\theta})$ respects the products, the algebraic Steenrod operations defined by $\tilde{\theta}$ satisfy the Cartan formula $P^{i}(x y)=\sum_{j+i=i} P^{j}(x) P^{k}(y), x, y \in$ $H^{*} A$.

\subsubsection{Review of the construction of Steenrod operations}

We consider the standard small free resolution of $\pi=\left\{1, \tau, \ldots, \tau^{p-1}\right\}$ :

$$
\begin{gathered}
W=\left(W_{i}\right)_{i \geqslant 0} ; \quad W_{i}=e_{i} \mathbb{F}_{p}[\pi] ; \quad W_{0} \simeq \mathbb{F}_{p}[\pi], \\
W_{i} \stackrel{\delta_{i}}{\longrightarrow} W_{i-1}, \quad \delta\left(e_{2 i+1}\right)=(1+\tau) e_{2 i}, \quad \delta\left(e_{2 i}\right)=(1+\tau+\cdots+\tau) e_{2 i-1}, \\
W \stackrel{\varepsilon_{W}}{\longrightarrow} \mathbb{K} \\
e_{i} \longmapsto \varepsilon_{W}\left(e_{i}\right)= \begin{cases}0 & \text { if } i \geqslant 0 \\
1_{\mathbb{K}} & \text { if } i=0 .\end{cases}
\end{gathered}
$$

Note that this standard free resolution equipped with its diagonal approximation $\psi_{W}$ has a coalgebra structure.

Let $\theta_{\pi}: W \otimes_{\pi} A^{\otimes p} \longrightarrow A$ be the map induced by the structural map $\theta$ and denote by $\theta^{*}$ the homomorphism $H\left(\theta_{\pi}\right)$. Observe that any section $\rho$ of a natural projection $A \cap k e r d \longrightarrow H(A)$ lifts to a $\pi$-linear chain map $\rho: W \otimes(H A)^{\otimes p} \longrightarrow W \otimes A^{\otimes p}$ and thus to a chain map $\rho_{\pi}: W \otimes_{\pi}(H A)^{\otimes p} \longrightarrow W \otimes_{\pi} A^{\otimes p}$. Since $W$ is a semifree $\mathbb{F}_{p^{-}}$ module in the sense of $[7], \rho^{*}=H\left(\rho_{\pi}\right)$ is an isomorphism. The algebraic Steenrod operations are defined as follows [15]; for $x \in H^{n}(A)$, each $e_{k} \otimes x^{\otimes p}$ is a cocycle in $W \otimes_{\pi}(H A)^{\otimes p}$. 
If $p=2$,

$$
\begin{aligned}
S q^{i}(x) & =\theta^{*} \circ \rho^{*}\left(\operatorname{cl}\left(e_{n-i} \otimes_{\pi} x^{\otimes p}\right)\right) \\
& =\operatorname{cl}\left(\theta_{\pi} \circ \rho_{\pi}\left(e_{n-i} \otimes_{\pi} x^{\otimes p}\right)\right) \\
& =\operatorname{cl}\left(\theta_{\pi}\left(e_{n-i} \otimes_{\pi} \rho_{\pi}\left(x^{\otimes p}\right)\right)\right) \\
& =\operatorname{cl}\left(\theta_{\pi}\left(e_{n-i} \otimes_{\pi} \rho_{\pi}(x)^{\otimes p}\right)\right) \\
& =\operatorname{cl}\left(\tilde{\theta}\left(\rho(x)^{\otimes p}\right)\right)\left(e_{n-i}\right)
\end{aligned}
$$

and if $p$ is odd,

$$
\begin{aligned}
P^{i}(x) & =(-1)^{i} \nu(n) \theta^{*} \circ \rho^{*}\left(\operatorname{cl}\left(e_{(n-2 i)(p-1)-1} \otimes x^{\otimes p}\right)\right) \\
& =(-1)^{i} \nu(n) \operatorname{cl}\left(\tilde{\theta}\left(\rho(x)^{\otimes p}\right)\right)\left(e_{(n-2 i)(p-1)}\right) \\
\beta P^{i}(x) & =(-1)^{i} \nu(n) \operatorname{cl}\left(\tilde{\theta}\left(\rho(x)^{\otimes p}\right)\right)\left(e_{(n-2 i)(p-1)-1}\right)
\end{aligned}
$$

where $\nu(n)=(-1)^{j}\left(\left(\frac{p-1}{2}\right) !\right)^{\epsilon}$ if $n=2 j+\epsilon, \epsilon=0,1$ and $\tilde{\theta}$ the $\pi$-chain map defined in Proposition 2.1.

2.1.3. Hochschild homology and negative cyclic homology

Here, Hochschild homology and negative cyclic homology are recalled.

Let $D A$ and $D C$ denote respectively the category of connected cochain algebras and the category of connected cochain coalgebras. The reduced bar and cobar construction are a pair of adjoint functors $B: D A \leftrightarrow D C: \Omega$ (see [6]). The generators of $B A$ (resp. $\Omega C$ ) are denoted $\left[a_{1}\left|a_{2}\right| \cdots \mid a_{k}\right] \in B_{k} A$ (resp. $\left\langle c_{1}\left|c_{2}\right| \cdots \mid c_{l}\right\rangle \in \Omega_{l} C$ ) and []$=1 \in B_{0} A \simeq \mathbb{K}\left(\right.$ resp. \langle\rangle$\left.=1 \in \Omega_{0} C \simeq \mathbb{K}\right)$.

The adjunction mentioned above yields for a cochain algebra $\left(A, d_{A}\right)$, a natural quasi-isomorphism of cochain algebras $\alpha_{A}: \Omega B A \longrightarrow A$ [17]. The linear map $\iota_{A}: A \longrightarrow \Omega B A$ such that $\iota_{A}(1)=1, \iota_{A}(a)=\langle[a]\rangle, a \in \bar{A}$ is a chain complex quasiisomorphism. In any case, it satisfies $\alpha_{A} \circ \iota_{A}=i d_{A}, i d_{\Omega B A}-\iota_{A} \circ \alpha_{A}=d_{\Omega B A} \circ h+$ $h \circ d_{\Omega B A}$ for some chain homotopy $h: \Omega B A \rightarrow \Omega B A$ such that $\alpha_{A} \circ h=0, h \circ \iota_{A}=0$, $h^{2}=0$.

Let $\left(A, d_{A}\right)$ be a cochain algebra. Recall also that the normalized Hochschild chain complex of $\left(A, d_{A}\right)$ is a graded vector space $\left\{\mathfrak{C}_{k} A\right\}_{k \geqslant 0}, \mathfrak{C}_{k} A=A \otimes B_{k} A$ where the generators of $\mathfrak{C}_{k} A$ are of the form $a_{0}\left[a_{1}\left|a_{2}\right| \ldots \mid a_{k}\right]$ if $k>0$ and $a[]$ if $k=0$. We set $\epsilon_{i}=\left|a_{0}\right|+\left|s a_{1}\right|+\left|s a_{2}\right|+\cdots+\left|s a_{i-1}\right|, i \geqslant 1$ and define the Hochschild differential $d=d^{1}+d^{2}$ by

$$
\begin{aligned}
d^{1}\left(a_{0}\left[a_{1}|\cdots| a_{k}\right]\right) & =d_{A}\left(a_{0}\right)\left[a_{1}\left|a_{2}\right| \cdots \mid a_{k}\right]-\sum_{i}=1^{k}(-1)_{i}^{\epsilon} a_{0}\left[a_{1}\left|a_{2}\right| \cdots\left|d_{A}\left(a_{i}\right)\right| \cdots \mid a_{k}\right] \\
d^{2}\left(a_{0}\left[a_{1}|\cdots| a_{k}\right]\right) & =(-1)^{\left|a_{0}\right|} a_{0} a_{1}\left[a_{2}|\cdots| a_{k}\right] \\
& +\sum_{i=2}^{k} a_{0}\left[a_{1}\left|a_{2}\right| \cdots\left|a_{i-1} a_{i}\right| \cdots \mid a_{k}\right] \\
& -(-1)^{\left|s a_{k}\right| \epsilon_{k}} a_{k} a_{0}\left[a_{2}|\cdots| a_{k}\right] .
\end{aligned}
$$

The Hochschild differential decreases the degree by one (see [13] or [20] for more details). 
By definition,

$$
H H_{*} A:=H_{*} \mathfrak{C} A
$$

is the Hochschild homology of the cochain algebra $\left(A, d_{A}\right)$. It is clear that $\mathfrak{C} A$ is concentrated in non-negative total degrees. Hence so is $H H_{*} A$.

If $\left(A, d_{A}\right)=\left(N^{*}(X ; \mathbb{K}), d_{N^{*}(X ; \mathbb{K})}\right)$ is the algebra of normalized singular cochains on the topological space $X$, then $\mathfrak{C}_{*} N^{*}(X ; \mathbb{K})$ is the normalized Hochschild chain complex of $X$ and $H H_{*} X:=H H_{*} N^{*}(X ; \mathbb{K})$ is the Hochschild homology of $X$.

For the cochain algebra $\left(A, d_{A}\right)$, the Connes operator is the linear map

$$
B: \mathfrak{C}_{*} A \rightarrow \mathfrak{C}_{*+1} A
$$

defined by $B a_{0}\left[a_{1}|\cdots| a_{n}\right]=\sum_{i=0}^{n}(-1)^{\epsilon_{i}} 1\left[a_{i}|\cdots| a_{n}\left|a_{0}\right| \cdots \mid a_{i-1}\right]$, where $\epsilon_{i}=\left|a_{0}\right|+$ $\left(\left|a_{0}\right|+\left|a_{1}\right|+\cdots+\left|a_{i-1}\right|+i\right)\left(\left|a_{i}\right|+\cdots+\left|a_{n}\right|+n-i+1\right)$. Consider the polynomial algebra $\mathbb{K}[u]$ on the single generator $u$ of upper degree +2 and form the complex $C_{*}^{-} A=\mathbb{K}[u] \otimes \mathfrak{C}_{*} A$ with differential $\mathfrak{D}$ defined by $\mathfrak{D}\left(u^{l} \otimes a_{0}\left[a_{1}|\cdots| a_{n}\right]\right)=u^{l} \otimes$ $d\left(a_{0}\left[a_{1}|\cdots| a_{n}\right]\right)+u^{l+1} \otimes B\left(a_{0}\left[a_{1}|\cdots| a_{n}\right]\right)$. The chain complex $C_{*}^{-} A$ is the negative cyclic chain complex of the cochain algebra $\left(A, d_{A}\right)$ (see $\left.[\mathbf{1 0}]\right)$. Let $L$ and $M$ be two graded $\mathbb{F}_{p}$-modules; $L \hat{\otimes} M$ will denote the tensor product defined by $(L \hat{\otimes} M)_{n}=$ $\prod L_{i} \otimes M_{n-i}$. Generally for a differential graded algebra $A, C_{*}^{-} A=\mathbb{F}_{p}[u] \hat{\otimes}_{\mathbb{F}_{p}} A$. So, for example, an element of degree $d$ is given by an infinite sum of the form $\sum u^{i} \otimes e_{i}$ where $e_{i} \in A_{d+2 i}[\mathbf{3}]$. If $A$ is positively graded, $C_{*}^{-} A=\mathbb{F}_{p}[u] \hat{\otimes}_{\mathbb{F}_{p}} A \cong \mathbb{F}_{p}[u] \otimes_{\mathbb{F}_{p}} A$, and its homology $H C^{-} A$ is the negative cyclic homology of $\left(A, d_{A}\right)$.

Again, it is clear that $C_{*}^{-} A$ is concentrated in non-negative total degrees and so is $H C_{*}^{-} A$.

If $\left(A, d_{A}\right)=N^{*}(X ; \mathbb{K})$ is the algebra of normalized singular cochains on the topological space $X$, then $C_{*}^{-} N^{*}(X ; \mathbb{K})$ is the negative cyclic chain complex of $X$, and $H C^{-} X:=H C_{*}^{-} N^{*}(X ; \mathbb{K})$ the associated negative cyclic homology.

If $\left(A ; d_{A}\right)$ is commutative (in the graded sense), then the multiplication $m_{A}: A \otimes$ $A \rightarrow A$ is a homomorphism of $D G$-algebras. Thus the composite $\mathfrak{C} m_{A} \circ s h: \mathfrak{C} A \otimes$ $\mathfrak{C} A \rightarrow \mathfrak{C} A$ defines a multiplication on $\mathfrak{C} A$ which makes it into a commutative algebra [13, 4.2.2], where $s h: \mathfrak{C} A \otimes \mathfrak{C} A \rightarrow \mathfrak{C}(A \otimes A)$ denotes the shuffle map.

\subsubsection{Homotopy}

1. Recall that $f, g \in \mathrm{D} A\left(A, A^{\prime}\right)$ are homotopic in $\mathrm{D} A$ if there exists a linear map $h: A \rightarrow A^{\prime}$ such that $f-g=d_{A^{\prime}} \circ h+h \circ d_{A}$ and $h(x y)=h(x) g(y)+$ $(-1)^{|x|} f(x) h(y)$ with $x, y \in A$. If $f, g \in \mathrm{D} A\left(A, A^{\prime}\right)$ are homotopic, we write $f \simeq{ }_{D A} g$.

2. Let $\left(A, d_{A}\right)$ (resp. $\left.\left(C, d_{C}\right)\right)$ be a differential graded algebra (resp. coalgebra).

Let $T(C, A)=\left\{t \in \operatorname{Hom}^{1}(C, A): D t=t \cup t, t \circ \eta_{C}=0=\varepsilon_{A} \circ t\right\}$ be the twisting cochain space as in $[\mathbf{1 7}, 1.8]$, where $D$ denotes the differential in $\operatorname{Hom}(C, A)$ and $\cup$ the usual cup-product on $\operatorname{Hom}(C, A)$.

The universal twisting cochains $t, t^{\prime} \in T(C, A)$ are homotopic in $T C(C, A)$ if there exists a linear map $h \in \operatorname{Hom}^{1}(C, A)$ such that $D h=t \cup h-h \cup t, h \circ \eta_{C}=$ $\eta_{A}$ and $\varepsilon A \circ h=\varepsilon_{C}$ and we write $t \simeq_{T} t^{\prime}[\mathbf{1 7}, 1.11]$.

3 . Denote by $\pi$-DM the category whose objects are differential graded modules over $\mathbb{F}_{p}$ equipped with an action of the cyclic group $\pi$ and whose morphisms are 
$\mathbb{F}_{p}[\pi]$-linear. If the $\mathbb{F}_{p}[\pi]$-linear maps $f$ and $g$ are homotopic with a $\mathbb{F}_{p}[\pi]$-linear homotopy, we write $f \simeq_{\pi-D M} g$.

Denote by $\pi$-DA the subcategory whose objects are differential graded algebras over $\mathbb{F}_{p}$ equipped with an action of the cyclic group $\pi$ and whose morphisms are linear morphisms of $\mathbb{F}_{p}[\pi]$-differential graded algebras. If the maps $f, g \in \pi$-DA are homotopic with a $\mathbb{F}_{p}[\pi]$-linear homotopy, we write $f \simeq_{\pi-D A} g$.

Lemma 2.2. Let $\left(T V, d_{V}\right)$ be a differential graded algebra and assume that a finite group $\pi$ acts freely on $V$. Let $\left(A, d_{A}\right)$ be a $\mathbb{F}_{p}[\pi]$-differential graded algebra and $f, g \in \pi-D A(T V, A)$. if $f \simeq_{\pi-D A} g$ then $C^{-} f \simeq_{\pi-D M} C^{-} g$.

Proof. We consider as in $[\mathbf{2 1}$, Lemma A.6], the cylinder object $I(T V, d):=$ $\left(T\left(V_{0} \oplus V_{1} \oplus s V\right)\right)$ on $(T V, d)$ :

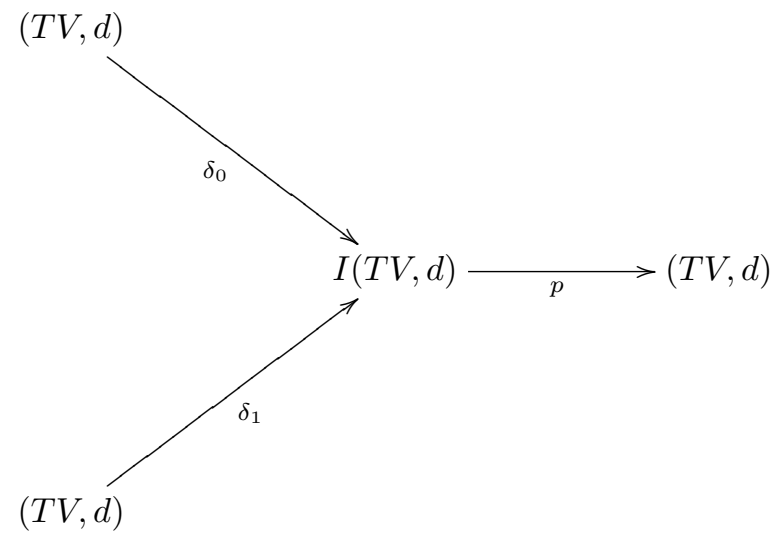

with $\delta_{0}(V)=V_{0}, \delta_{1}(V)=V_{1}, p\left(v_{0}\right)=p\left(v_{0}\right)=v, p\left(s v_{0}\right)=0, D=d$ on $V_{0}$ and $V_{1}, D s v=d S v$ where $S$ is the unique $\left(\delta_{0}, \delta_{1}\right)$-derivation $S: T V \longrightarrow T\left(V_{0} \oplus V_{1} \oplus\right.$ $s V$ ) extending the graded isomorphism $s: V \longrightarrow s V$. The free $\pi$-action on $T V$ naturally extends to a free $\pi$-action on $I(T V, d)$ so that $I(T V, d)$ is a $\pi$-free algebra and the maps $p, \delta_{0}, \delta_{1}$ are $\pi$-equivariant quasi-isomorphisms of differential graded algebras. Moreover from the free $\pi$-action on the cylinder object $I(T V, d)$, we have a free $\pi$-action on the negative cyclic complex $C_{*}^{-} I(T V)$ of the cylinder object $I(T V)$ by the following rule:

For any $u^{l} \otimes x_{0}\left[x_{1}\left|x_{2}\right| \cdots\left|x_{n-1}\right| x_{n}\right] \in C_{*}^{-} I(T V)$ and $\sigma \in \pi$,

$$
\begin{aligned}
\sigma \cdot u^{l} \otimes x_{0}\left[x_{1}\left|x_{2}\right| \cdots\left|x_{n-1}\right| x_{n}\right] & =u^{l} \otimes \sigma . \\
& x_{0}\left[\sigma \cdot x_{1}\left|\sigma \cdot x_{2}\right| \cdots\left|\sigma \cdot x_{n-1}\right| \sigma \cdot x_{n}\right] .
\end{aligned}
$$

Thus $C_{*}^{-} I(T V)$ is an object of the category $\pi$-DM.

By definition, $f \simeq_{D A} g$ (resp. $f \simeq_{\pi-D A} g$ ) if and only if there exists $H \in$ $D A(I(T V), A)($ resp. $H \in \pi-D A(I(T V), A))$ such that $H \circ \delta_{0}=f$ and $H \circ$ $\delta_{1}=g$.

If $H \in \pi$-DA( ( (TV)) is a homotopy between $f$ and $g$, then $C_{*}^{-} H$ is a $\pi$-linear homotopy between $C_{*}^{-} f$ and $C_{*}^{-} g$. Hence $C_{*}^{-} f \simeq_{\pi-D M} C_{*}^{-} g$. 
2.1.5. A strongly homotopy commutative algebra (see $[\mathbf{1 7}, 4]$ )

A strongly homotopy commutative algebra (shc-algebra for short) is a triple ( $A, d_{A}$, $\left.\mu_{A}\right)$ with $\left(A, d_{A}\right) \in \operatorname{Obj} D A$ and $\mu_{A} \in D A(\Omega B(A \otimes A), \Omega B A)$ satisfying

1. $\alpha_{A} \circ \mu_{A} \circ \iota_{A \otimes A}=m_{A}$, where $m_{A}$ is the product on $A$;

2. $\alpha_{A} \circ \mu_{A} \circ \Omega B\left(i d_{A} \otimes \eta_{A}\right) \circ \iota_{A}=\alpha_{A} \circ \mu_{A} \circ \Omega B\left(\eta_{A} \otimes i d_{A}\right) \circ \iota_{A}=i d_{A}$, where $\mathbb{K}$ $\stackrel{\eta_{A}}{\longrightarrow} A$ is the unit in $A$; i.e. $\eta_{A}$ is the unit up to homotopy for $\mu_{A}$;

3. $\mu_{A} \circ \Omega B\left(\alpha_{A} \otimes i d_{A}\right) \circ \Omega B\left(\mu_{A} \otimes i d_{A}\right) \circ \chi_{(A \otimes A) \otimes A} \simeq_{D A} \mu_{A} \circ \Omega B\left(i d_{A} \otimes \alpha_{A}\right) \circ$ $\Omega B\left(i d_{A} \otimes \mu_{A}\right) \circ \chi_{A \otimes(A \otimes A)}$; i.e. $\mu_{A}$ is associative up to homotopy;

4. $\mu_{A} \circ \Omega B T \simeq_{D A} \mu_{A}$; where $T$ denotes the interchange map $T(x \otimes y)=$ $(-1)^{|x||y|} y \otimes x$; i.e. $\mu_{A}$ is commutative up to homotopy.

The following natural homomorphisms of DG-algebras are defined in $[\mathbf{1 7}, 2.2]$;

$\Omega B(\Omega B(A \otimes A) \otimes A) \stackrel{\chi(A \otimes A) \otimes A}{\longleftrightarrow} \Omega B(A \otimes A \otimes A) \stackrel{\chi_{A \otimes(A \otimes A)}}{\longrightarrow} \Omega B(A \otimes \Omega B(A \otimes A))$, and satisfy

$$
\alpha_{(A \otimes A) \otimes A} \circ \chi_{(A \otimes A) \otimes A}=\alpha_{A \otimes A \otimes A}=\alpha_{A \otimes(A \otimes A)} \circ \chi_{A \otimes(A \otimes A)} .
$$

Consider $A$ and $A^{\prime}$ in $\operatorname{Obj} D A$. The map $f \in D A\left(A, A^{\prime}\right)$ is a shc-map from $\left(A, d_{A}\right.$, $\left.\mu_{A}\right)$ to $\left(A^{\prime}, d_{A}^{\prime}, \mu_{A^{\prime}}\right)$ if

1. $\alpha_{A^{\prime}} \circ \Omega B f \circ \iota_{A}=f$;

2. $\alpha_{A^{\prime}} \circ \Omega B f \circ \eta_{\Omega B A}=\eta_{A^{\prime}}$;

3. $\Omega B f \circ \mu_{A} \simeq_{D A} \mu_{A}^{\prime} \circ \Omega B(f \otimes f)$.

As proved by [17], an example of shc-cochain algebra is the algebra $N^{*}(X ; \mathbb{K})$ of normalized singular cochains of a topological space $X$.

On the other hand it is proved in $[\mathbf{2 0}]$ that if $\left(A, d_{A}, \mu_{A}\right)$ is a shc-cochain algebra, then

1. $B A$ is a differential graded Hopf algebra and $H^{*} B A$ is a commutative graded Hopf algebra;

2. $\mathfrak{C} A$ is a (non associative) graded algebra such that $H H_{*} A$ is a commutative graded algebra;

3. if $f:\left(A, d_{A}, \mu_{A}\right) \rightarrow\left(A^{\prime}, d_{A}^{\prime}, \mu_{A^{\prime}}\right)$ is a morphism of shc-cochain algebras, we have $A \stackrel{i}{\longrightarrow} \quad \mathfrak{C} A \stackrel{\rho}{\longrightarrow} B A$ the commutative diagram $f \downarrow \quad \downarrow \mathfrak{C} f \quad \downarrow B f$, where $i, i^{\prime}, \rho$,

$$
A^{\prime} \stackrel{i^{\prime}}{\longrightarrow} \quad \mathfrak{C} A^{\prime} \quad \stackrel{\rho^{\prime}}{\longrightarrow} \quad B A^{\prime}
$$

$\rho^{\prime}$ and $\mathfrak{C} f$ are homomorphisms of cochain algebras and $B f$ is a homomorphism of differential graded Hopf algebras.

Remark 2.3. We recall the following facts given in [21, A.2]:

1- If $\left(\left(A, d_{A}\right), \mu_{A}\right)$ is a shc differential graded algebra, so is $\Omega B(A)$, with the shc structural map $\mu_{\Omega B(A)}$ given by the composite $\mu_{\Omega B(A)}=\theta_{\Omega B(A)} \circ \mu_{A} \circ \Omega B\left(\alpha_{A}\right.$ $\left.\otimes \alpha_{A}\right)$, where $\theta_{\Omega B(A)} \in D A(\Omega B(A), \Omega B \Omega B(A))$ is the unique section of $\alpha_{\Omega B(A)}$ $\in D A(\Omega B \Omega B(A), \Omega B(A))$ such that $\alpha_{A} \circ \alpha_{\Omega B(A)} \circ \theta_{\Omega B(A)}=\alpha_{A}$. 
2- If $\left(\left(A, d_{A}\right), \mu_{A}\right)$ is a shc differential graded algebra and $\left(\left(W, \delta_{W}\right), \psi_{W}\right)$ a standard small free resolution of $\pi$ equipped with its differential graded coalgebra structure, then $\operatorname{Hom}(W ; A)$ is a shc differential graded algebra with the shc structural map $\mu_{\mathrm{Hom}(W ; A)} \in D A\left(\Omega B\left([\operatorname{Hom}(W ; A)]^{\otimes 2}\right), \Omega B([\operatorname{Hom}(W ; A)])\right)$ defined by

$$
\mu_{\mathrm{Hom}(W ; A)}=\Omega B\left(\operatorname{Hom}\left(W, \alpha_{A} \circ \mu_{A}\right)\right) \circ \theta_{\operatorname{Hom}\left(W ; A^{\otimes 2}\right)} \circ \Omega B\left(\psi_{A}\right),
$$

where $[\operatorname{Hom}(W ; A)]^{\otimes 2} \stackrel{\psi_{A}}{\longrightarrow}\left[\operatorname{Hom}\left(W ; A^{\otimes 2}\right)\right]$ the map defined by $\psi_{A}(f \otimes g)=$ $(f \otimes g) \circ \psi_{W}$ is the homomorphism of differential graded algebras satisfying $\operatorname{Hom}\left(W, m_{A}\right) \circ \psi_{A}=\cup$ and $\theta_{\operatorname{Hom}\left(W, A^{\otimes 2}\right)} \in D A(\Omega B \operatorname{Hom}(W, A \otimes A), \Omega B($ Hom $(W, \Omega B(A \otimes A))))$ the unique homomorphism of differential graded algebras such that

$$
\operatorname{Hom}\left(W, \alpha_{A \otimes 2}\right) \circ \alpha_{\operatorname{Hom}\left(W, \Omega B\left(A^{\otimes 2}\right)\right)} \circ \theta_{\operatorname{Hom}\left(W, A^{\otimes 2}\right)}=\alpha_{\operatorname{Hom}(W, A \otimes A)} .
$$

2.1.6. Shc-equivalence and shc-formality $[\mathbf{2 0}, 5]$

The shc cochain algebras $\left(A, d_{A}, \mu_{A}\right)$ and $\left(A^{\prime}, d_{A^{\prime}}, \mu_{A^{\prime}}\right)$ are said to be $s h c$-equivalent $\left(A \simeq_{s h c} A^{\prime}\right)$ if there exists a sequence of shc morphisms $A \leftarrow A_{1} \rightarrow \cdots \rightarrow A^{\prime}$ which are quasi-isomorphisms. One particular case of $s h c$-equivalence is the $s h c$-formality. Recall that the cohomology algebra of a $s h c$ cochain algebra is commutative. Every commutative cochain algebra is a shc algebra with shc structural map $\mu_{A}=\Omega B\left(m_{A}\right): \Omega B(A$ $\otimes A) \rightarrow \Omega B A$, where $m_{A}$ is the product on $A$.

A shc cochain algebra $A$ is $s h c$-formal if it is shc-equivalent to its cohomology algebra $H^{*} A$.

2.1.7. A $\pi$-strongly homotopy commutative algebra; see $[\mathbf{2 1}, 1.6]$

Let $\left(A, d_{A}, \mu_{A}\right)$ and $\left(A^{\prime}, d_{A^{\prime}}, \mu_{A^{\prime}}\right)$ be two shc differential graded algebras. There exists a natural homomorphism $\mu_{A \otimes A^{\prime}} \in D A\left(\Omega B\left(\left(A \otimes A^{\prime}\right)^{\otimes 2}\right) ; \Omega B\left(A \otimes A^{\prime}\right)\right)$ such that $(A$ $\left.\otimes A^{\prime}, d_{A \otimes A^{\prime}} ; \mu_{A \otimes A^{\prime}}\right)$ is a shc differential graded algebra.

In particular, if $\left(A, d_{A}, \mu_{A}\right)$ is a shc differential graded algebra, then for any $n \geqslant 2$, there exists a homomorphism of differential graded algebras called the shc iterated structural map $\Omega B\left(A^{\otimes n}\right) \stackrel{\mu_{A}^{(n)}}{\longrightarrow} \Omega B(A)$ such that $\mu^{(2)}=\mu$ and $\alpha_{A} \circ \mu^{(n)} \circ i_{A} \otimes n \simeq m_{A}^{(n)}$ (see [21, Lemma A.3].

A $s h c$-algebra $\left(A, d_{A}, \mu_{A}\right)$ is a $\pi$-strongly homotopy commutative algebra (a $\pi$-shcalgebra for short) if there exists a map $\Omega B\left(A^{\otimes p}\right) \stackrel{\tilde{\kappa}_{A}}{\longrightarrow} \operatorname{Hom}(W, A)$ that is a $\pi$-linear homomorphism of differential graded algebras such that

$$
e v_{0} \circ \tilde{\kappa}_{A} \simeq_{D A} \alpha_{A} \circ \mu_{A}^{(p)},
$$

where $p$ is the prime number characteristic of $\mathbb{F}_{p}$.

The action of $S_{p}$ on $B\left(A^{\otimes p}\right)$ (resp. $\Omega B\left(A^{\otimes p}\right)$ ) is defined by the rule: For any $\sigma \in S_{p}$ $\sigma\left[a_{1}\left|a_{2}\right| \cdots\left|a_{p-1}\right| a_{p}\right]=\left[a_{\sigma(1)}\left|a_{\sigma(2)}\right| \cdots\left|a_{\sigma(p-1)}\right| a_{\sigma(p)}\right], a_{i} \in A^{\otimes p} \quad\left(\operatorname{resp} . \quad \sigma<x_{1}\left|x_{2}\right| \cdots\right.$ $\left.\left|x_{p-1}\right| x_{p}>=<\sigma x_{1}\left|\sigma x_{2}\right| \cdots\left|\sigma x_{p-1}\right| \sigma x_{p}>, x_{i} \in B\left(A^{\otimes p}\right)\right)$.

Recall that a strict $\pi$-shc homomorphism $\left(\left(A, d_{A}\right), \mu_{A}, \tilde{\kappa}_{A}\right) \stackrel{f}{\longrightarrow}\left(\left(A^{\prime}, d_{A^{\prime}}\right), \mu_{A^{\prime}}, \tilde{\kappa}_{A^{\prime}}\right)$ is a strict $s h c$ homomorphism such that the following homomorphisms of differential graded algebras $\tilde{\kappa}_{A^{\prime}} \circ \Omega B\left(f^{\otimes p}\right)$ and $\operatorname{Hom}(W, f) \circ \tilde{\kappa}_{A}$ are $\pi$-linear homotopic. 


\section{Proof of the first part of Theorem 1.1}

As explained in $[\mathbf{1 3}, 4.3]$, a $(p, q)$-cyclic shuffle is a permutation $\{\sigma(1), \ldots, \sigma(p), \sigma(p$ $+1), \ldots, \sigma(p+q)\}$ in $S_{p+q}$ obtained as follows: Perform a cyclic permutation of any order on the set $\{1, \ldots, p\}$ and perform a cyclic permutation of any order on the set $\{p+1, \ldots, p+q\}$. We shuffle the two results to obtain $\{\sigma(1), \ldots \sigma(p), \sigma(p+$ $1), \ldots, \sigma(p+q)\}$ in $S_{p+q}$. The permutation obtained in that way is a cyclic shuffle if 1 appears before $p+1$; we denote by $\sum_{p, q}^{C}$ the set of $(p, q)$-cyclic shuffles.

A map $\perp: \mathfrak{C}_{p}(A, A) \otimes \mathfrak{C}_{q}(A, A) \rightarrow \mathfrak{C}_{p+q}\left(A^{\otimes 2}, A^{\otimes 2}\right)$ is defined by

$$
\begin{aligned}
a_{0}\left[a_{1}\left|a_{2}\right| \cdots\left|a_{p-1}\right| a_{p}\right] & \perp b_{0}\left[b_{1}\left|b_{2}\right| \cdots\left|b_{q-1}\right| b_{q}\right] \\
& =\sum_{\sigma \in \sum_{(p, q)}^{C}}(-1)^{\varepsilon(\sigma)} a_{0} \otimes b_{0}\left[c_{\sigma(1)}\left|c_{\sigma(2)}\right| \cdots\left|c_{\sigma(p+q-1)}\right| c_{\sigma(p+q)}\right],
\end{aligned}
$$

where $\varepsilon(\sigma)$ is the signature of $\sigma$ and

$$
c_{\sigma(i)}= \begin{cases}a_{\sigma(i)} \otimes 1 & \text { if } 1 \leqslant i \leqslant p \\ 1 \otimes b_{\sigma(i)-p} & \text { if } p+1 \leqslant i \leqslant p+q .\end{cases}
$$

The cyclic shuffle (see $[\mathbf{1 3}, 4.3 .2]$ ) is a linear map

$$
\mathfrak{C}_{*}(A, A) \otimes \mathfrak{C}_{*^{\prime}}(A, A) \stackrel{s h^{\prime}}{\longrightarrow} \mathfrak{C}_{*+*^{\prime}+2}\left(A^{\otimes 2}, A^{\otimes 2}\right)
$$

defined by

$$
\begin{aligned}
\operatorname{sh}^{\prime}\left(a_{0}\left[a_{1}\left|a_{2}\right| \cdots\left|a_{p-1}\right| a_{p}\right] \otimes b_{0}\left[b_{1}\left|b_{2}\right| \cdots\left|b_{q-1}\right| b_{q}\right]\right) & \\
= & 1\left[a_{0}\left|a_{1}\right| a_{2}|\cdots| a_{p-1} \mid a_{p}\right] \perp 1\left[b_{0}\left|b_{1}\right| b_{2}|\cdots| b_{q-1} \mid b_{q}\right] .
\end{aligned}
$$

It is clear from the definition that if $a_{0}=1$ or $b_{0}=1$ then

$$
\operatorname{sh}^{\prime}\left(a_{0}\left[a_{1}\left|a_{2}\right| \cdots\left|a_{p-1}\right| a_{p}\right] \otimes b_{0}\left[b_{1}\left|b_{2}\right| \cdots\left|b_{q-1}\right| b_{q}\right]\right)=0 .
$$

Proposition 3.1. (see $[13,4.3 .7])$ The following identities are satisfied:

- $d \circ s h=s h \circ d$;

- $s h^{\prime} \circ B=B \circ s h^{\prime}$;

- $B \circ s h-s h \circ B+d \circ s h^{\prime}-s h^{\prime} \circ d=0$,

where $d$ and $B$ are the Hochschild differential and the Connes operator respectively. When $\mathbb{K}[u] \otimes \mathfrak{C}(A) \otimes \mathfrak{C}(A)$ and $\mathbb{K}[u] \otimes \mathfrak{C}(A \otimes A)$ are equipped with the obvious differentials denoted respectively by $\mathcal{D}$ and $\hat{\mathcal{D}}$, the linear map $S h: \mathbb{K}[u] \otimes \mathfrak{C}(A) \otimes \mathfrak{C}(A) \longrightarrow$ $\mathbb{K}[u] \otimes \mathfrak{C}(A \otimes A)$ defined by $S h=i d_{\mathbb{K}[u]} \otimes s h+u\left(i d_{K}[u] \otimes s h^{\prime}\right)$ satisfies $\mathcal{D} \circ S h=$ $S h \circ \hat{\mathcal{D}}$.

Let $\left(A, d_{A}, \mu_{A}\right)$ be a shc-algebra. The chain map $m_{C_{*}^{-} A}$ given by the composite

$$
\begin{aligned}
& \mathbb{K}[u] \otimes \mathfrak{C}(A) \otimes \mathbb{K}[u] \otimes \mathfrak{C}(A) \stackrel{i d \otimes T \otimes i d}{\longrightarrow} \mathbb{K}[u] \otimes \mathbb{K}[u] \otimes \mathfrak{C}(A) \otimes \mathfrak{C}(A) \stackrel{m_{\mathbb{K}[u]} \otimes i d}{\longrightarrow} \\
& \mathbb{K}[u] \otimes \mathfrak{C}(A) \otimes \mathfrak{C}(A) \stackrel{S h}{\longrightarrow} \mathbb{K}[u] \otimes \mathfrak{C}(A \otimes A) \stackrel{S_{A \otimes A}}{\longrightarrow} \\
& \mathbb{K}[u] \otimes \mathfrak{C}(\Omega B(A \otimes A)) \stackrel{C_{*}^{-}\left(\mu_{A}\right)}{\longrightarrow} C_{*}^{-}(\Omega B(A)) \stackrel{C_{*}^{-}\left(\alpha_{A}\right)}{\longrightarrow} C_{*}^{-}(A)
\end{aligned}
$$


defined a product on $C_{*}^{-}(A)$ associative up to homotopy; where $S_{A \otimes A}$ is a linear section induced by the surjective quasi-isomorphism $C_{*}^{-}(\Omega B(A \otimes A)) \stackrel{C_{*}^{-}\left(\alpha_{A \otimes A}\right)}{\longrightarrow}$ $C_{*}^{-}(A \otimes A), T$ the interchange isomorphism and $m_{\mathbb{K}[u]}$ the product on $\mathbb{K}[u]$.

Precomposing $H_{*}\left(m_{C_{*}^{-}(A)}\right)$ by the Künneth isomorphism yields an associative product on $H C^{-}(A)$. Together with this product, $H C^{-}(A)$ is an associative graded algebra (see $[\mathbf{1 8}]$ ).

Proposition 3.2. Let $\left(\left(A ; d_{A}\right)\right)$ be a cochain algebra and $\left(\left(W ; \delta_{W}\right) ; \Psi_{W}\right)$ a standard free resolution of $\pi$ equipped with its coassociative coalgebra structure. There exists a natural homomorphism of chain complexes $\phi_{A}$ such that the following diagram commutes.

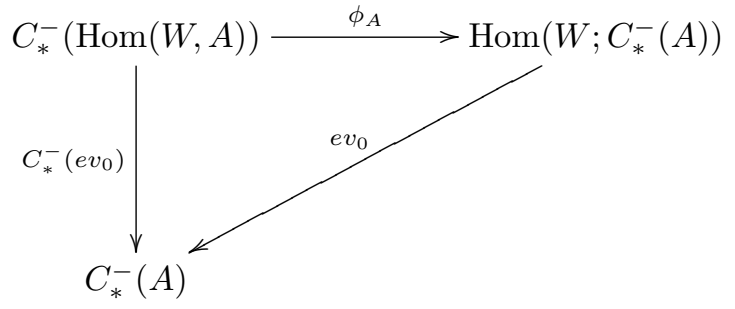

Proof. Let us prove the existence of the map $\phi_{A}$.

We define $\phi_{A}$ by

$$
\begin{array}{rll}
\phi_{A}: \mathbb{K}[u] \otimes \mathfrak{C}(\operatorname{Hom}(W ; A)) & \longrightarrow & \operatorname{Hom}(W ; \mathbb{K}[u] \otimes \mathfrak{C} A) \\
u^{l} \otimes f_{0}\left[f 1\left|f_{2}\right| \cdots\left|f_{k-1}\right| f_{k}\right] & \longmapsto & \phi_{A}\left(u^{l} \otimes f_{0}\left[f 1\left|f_{2}\right| \cdots\left|f_{k-1}\right| f_{k-1}\right]\right)
\end{array}
$$

such that

$$
\begin{aligned}
& \phi_{A}\left(u^{l} \otimes f_{0}\left[f 1\left|f_{2}\right| \cdots\left|f_{k-1}\right| f_{k-1}\right]\right) \\
& \quad=\left(I d \otimes I d \otimes s^{\otimes k}\right) \circ\left(g\left(u^{l}\right) \otimes f_{0} f 1 \otimes f_{2} \otimes \cdots \otimes f_{k-1} \otimes f_{k}\right) \circ \Psi_{W}^{(k+1)},
\end{aligned}
$$

where $W \stackrel{\Psi_{W}^{(1)}:=\Psi_{W}}{\longrightarrow} W \otimes W ; W \stackrel{\Psi_{W}^{(k+1)}}{\longrightarrow} W^{\otimes k+2}$ denotes the iterated diagonal, and $g$ the map defined by

$$
\begin{aligned}
\mathbb{K}[u] \stackrel{g}{\longrightarrow} \operatorname{Hom}(W ; \mathbb{K}[u]) \\
u^{l} \longmapsto g\left(u^{l}\right)
\end{aligned}
$$

such that

$$
\begin{aligned}
g\left(u^{l}\right): W \longrightarrow \mathbb{K}[u] & \\
e_{i} \longmapsto g\left(u^{l}\right)\left(e_{i}\right) & =g\left(u^{l}\right)\left(\tau^{j} e_{i}\right) \\
& = \begin{cases}u^{l-k} & \text { if } i=2 k, 0 \leqslant k \leqslant l ; \\
0 & \text { if not. }\end{cases}
\end{aligned}
$$


Here we check in detail that $\phi_{A}$ commutes with the differentials.

Consider for this purpose $\bar{D}$, the differential in $C_{*}^{-}(\operatorname{Hom}(W ; A))$ defined by

$$
\begin{aligned}
\bar{D}\left(u^{l} \otimes f_{0}\left[f_{1}\left|f_{2}\right| \cdots\left|f_{k-1}\right| f_{k}\right]\right) & =u^{l} \otimes d_{\mathfrak{C}_{*}(\operatorname{Hom}(W ; A))}\left(f_{0}\left[f_{1}\left|f_{2}\right| \cdots\left|f_{k-1}\right| f_{k-1}\right]\right) \\
& +u^{l+1} \otimes B\left(f_{0}\left[f_{1}\left|f_{2}\right| \cdots\left|f_{k-1}\right| f_{k-1}\right]\right)
\end{aligned}
$$

and $\tilde{D}$ the differential in $\operatorname{Hom}\left(W ; C_{*}^{-} A\right)$ defined by $\tilde{D}(f)=D \circ f-(-1)^{|f|} f \circ \delta$ where $D$ denotes the differential in $C_{*}^{-} A ; f \in \operatorname{Hom}\left(W ; C_{*}^{-} A\right)$.

We have to prove that $\tilde{D} \circ \phi_{A}=\phi_{A} \circ \bar{D}$.

$$
\begin{aligned}
\phi_{A} \circ \bar{D}\left(u^{l} \otimes f_{0}\left[f 1\left|f_{2}\right| \cdots\left|f_{k-1}\right| f_{k}\right]\right)= & \phi_{A}\left(u^{l} \otimes d_{\mathfrak{C}_{*}(\operatorname{Hom}(W ; A))}\left(f_{0}\left[f 1\left|f_{2}\right| \cdots\left|f_{k-1}\right| f_{k}\right]\right)\right. \\
& \left.+u^{l+1} \otimes B\left(f_{0}\left[f 1\left|f_{2}\right| \cdots\left|f_{k-1}\right| f_{k}\right]\right)\right) .
\end{aligned}
$$

From the definition of the Hochschild differential, one has

$$
\begin{aligned}
& \phi_{A}\left(u^{l} \otimes d^{1}\left(f_{0}\left[f_{1}|\cdots| f_{k}\right]\right)\right) \\
& =\phi_{A}\left[u^{l} \otimes d f_{0}\left[f_{1}|\cdots| f_{k}\right]-\sum_{i=1}^{k}(-1)^{\varepsilon_{i}} u^{l} \otimes f_{0}\left[f_{1}|\cdots| d\left(f_{i}\right)|\cdots| f_{k}\right]\right] \\
& =\phi_{A}\left(u^{l} \otimes d\left(f_{0}\right)\left[f_{1}|\cdots| f_{k}\right]\right)-\sum_{i=1}^{k}(-1)^{\varepsilon_{i}} \phi_{A}\left(u^{l} \otimes f_{0}\left[f_{1}|\cdots| d\left(f_{i}\right)|\cdots| f_{k}\right]\right) \\
& =\left(I d \otimes I d \otimes s^{\otimes k}\right) \circ\left(g\left(u^{l}\right) \otimes d f_{0} \otimes f_{1} \otimes \cdots \otimes f_{k}\right) \circ \Psi_{W}^{(k+2)}- \\
& \sum_{i=1}^{k}(-1)^{\varepsilon_{i}}\left(I d \otimes I d \otimes s^{\otimes k}\right)\left(g\left(u^{l}\right) \otimes f_{0} \otimes f_{1} \otimes \cdots \otimes d f_{i} \otimes \cdots \otimes\right. \\
& \left.f_{k}\right) \circ \Psi_{W}^{(k+2)} \\
& =\left(I d \otimes I d \otimes s^{\otimes k}\right)\left(g\left(u^{l}\right) \otimes\left(d_{A} f_{0}-(-1)^{\left|f_{0}\right|} f_{0} \delta_{W}\right) \otimes f_{1} \otimes \cdots \otimes f_{k}\right) \circ \Psi_{W}^{(k+2)}- \\
& \sum_{i=1}^{k}(-1)^{\varepsilon_{i}}\left(I d \otimes I d \otimes s^{\otimes k}\right)\left(g\left(u^{l}\right) \otimes f_{0} \otimes f_{1} \otimes\left(d_{A} f_{i}-(-1)^{\left|f_{i}\right|} f_{i} \circ \delta_{W}\right) \otimes \cdots\right. \\
& \left.\otimes f_{k}\right) \circ \Psi_{W}^{(k+2)} \\
& =\left(I d \otimes I d \otimes s^{\otimes k}\right)\left(I d \otimes d_{A} \otimes I d-I d \otimes I d \otimes d_{A^{\otimes k}}\right) \circ\left(g\left(u^{l}\right) \otimes f_{0} \otimes f_{1} \otimes \cdots\right. \\
& \left.\otimes f_{k}\right) \circ \Psi_{W}^{(k+2)}-(-1)^{\left(\sum_{0}^{k}\left|f_{i}\right|\right)+k}\left(I d \otimes I d \otimes s^{\otimes k}\right) \circ\left(g\left(u^{l}\right) \otimes f_{0} \otimes f_{1} \otimes \cdots \otimes f_{k}\right) \\
& \circ\left(I d \otimes \delta_{W \otimes k+1}\right) \circ \Psi_{W}^{k+1} \\
& =\left(I d \otimes d_{1}\right) \circ\left(I d \otimes I d \otimes s^{\otimes k}\right)\left(g\left(u^{l}\right) \otimes f_{0} \otimes f_{1} \otimes f_{2} \otimes \cdots \otimes f_{k-1} \otimes f_{k}\right) \circ \Psi_{W}^{(k+2)}- \\
& (-1)^{\varepsilon_{k}}\left(I d \otimes I d \otimes s^{\otimes k}\right)\left(g\left(u^{l}\right) \otimes f_{0} \otimes f_{1} \otimes \cdots \otimes f_{k}\right) \circ\left(I d \otimes \delta_{W}\right) \circ \varphi_{W}^{k+1} \\
& =\left(I d \otimes d^{1}\right) \circ \phi_{A}\left(u^{l} \otimes f_{0}\left[f_{1}|\cdots| f_{k}\right]\right)-(-1)^{\varepsilon_{k}} \phi_{A}\left(u^{l} \otimes f_{0}\left[f_{1}|\cdots| f_{k}\right]\right) \circ \delta .
\end{aligned}
$$


This result follows from the fact that $\left(\left(W, \delta_{W}\right), \psi_{W}\right)$ is a differential graded coalgebra:

$$
\begin{aligned}
& \phi_{A}\left(u^{l} \otimes d^{2}\left(f_{0}\left[f_{1}\left|f_{2}\right| \cdots\left|f_{k-1}\right| f_{k}\right]\right)\right) \\
& =\phi_{A}\left((-1)^{\left|f_{0}\right|} u^{l} \otimes\left(f_{0} \cup f_{1}\right)\left[f_{2}\left|f_{3}\right| \cdots\left|f_{k-1}\right| f_{k}\right]+\right. \\
& \sum_{i=2}^{k}(-1)^{\varepsilon_{i}} u^{l} \otimes f_{0}\left[f_{1}\left|f_{2}\right| \cdots\left|f_{i-1} \cup f_{i}\right| \cdots\left|f_{k-1}\right| f_{k}\right]- \\
& \left.(-1)^{\left(\left|f_{k}\right|+1\right) \varepsilon_{k}} u^{l} \otimes\left(f_{k} \cup f_{0}\right)\left[f_{1}\left|f_{2}\right| \cdots\left|f_{k-2}\right| f_{k-1}\right]\right) \\
& =\phi_{A}\left((-1)^{\left|f_{0}\right|} u^{l} \otimes m_{A} \circ\left(f_{0} \otimes f_{1}\right) \circ \Psi_{W}\left[f_{2}\left|f_{3}\right| \cdots\left|f_{k-1}\right| f_{k}\right]+\right. \\
& \sum_{i=2}^{k}(-1)^{\varepsilon_{i}} u^{l} \otimes f_{0}\left[f_{1}\left|f_{2}\right| \cdots\left|m_{A} \circ\left(f_{i-1} \otimes f_{i}\right) \circ \Psi_{W}\right| \cdots\left|f_{k-1}\right| f_{k}\right]- \\
& \left.(-1)^{\left(\left|f_{k}\right|+1\right) \varepsilon_{k}} u^{l} \otimes m_{A} \circ\left(f_{k} \otimes f_{0}\right) \circ \psi_{W}\left[f_{1}\left|f_{2}\right| \cdots\left|f_{k-2}\right| f_{k-1}\right]\right) \\
& =(-1)^{\left|f_{0}\right|}\left(I d \otimes I d \otimes s^{\otimes(k-1)}\right) \circ\left[g\left(u^{l}\right) \otimes m_{A} \circ\left(f_{0} \otimes f_{1}\right)\right. \\
& \left.\circ \psi_{W} \otimes f_{2} \otimes f_{3} \otimes \cdots \otimes f_{k-1} \otimes f_{k}\right] \circ \Psi_{W}^{(k)}+ \\
& \sum_{i=2}^{k}(-1)^{\varepsilon_{i}}\left(I d \otimes I d \otimes s^{\otimes(k-1)}\right) \circ \\
& {\left[g\left(u^{l}\right) \otimes f_{0} \otimes f_{1} \otimes \cdots \otimes m_{A} \circ\left(f_{i-1} \otimes f_{i}\right) \circ \Psi_{W} \otimes \cdots \otimes f_{k}\right] \circ \varphi_{W}^{k}-} \\
& (-1)^{\left(\left|f_{k}\right|+1\right) \varepsilon_{k}}\left(I d \otimes I d \otimes s^{\otimes(k-1)}\right) \\
& \circ\left[g\left(u^{l}\right) \otimes m_{A} \circ\left(f_{k} \otimes f_{0}\right) \circ \Psi_{W} \otimes f_{1} \otimes f_{2} \otimes \cdots \otimes f_{k-2} \otimes f_{k-1}\right] \\
& =\left(I d \otimes I d \otimes s^{\otimes(k-1)}\right) \circ\left[\left(I d \otimes m_{A} \otimes I d\right)+\right. \\
& \left.\sum_{2}^{k}(-1)^{i}\left(I d \otimes I d \otimes m_{A} \otimes I d\right)+\left(I d \otimes\left(m_{A} \otimes I d\right) \circ \sigma_{k}\right)\right] \circ \\
& \left(I d \otimes I d \otimes s^{\otimes(k-1)}\right)^{-1} \circ \phi_{A}\left(u^{l} \otimes f_{0}\left[f_{1}\left|f_{2}\right| \cdots\left|f_{k-1}\right| f_{k}\right]\right) \\
& =\left(I d \otimes d_{\mathfrak{C}(A)}^{2}\right) \circ \phi_{A}\left(u^{l} \otimes f_{0}\left[f_{1}\left|f_{2}\right| \cdots\left|f_{k-1}\right| f_{k}\right]\right) .
\end{aligned}
$$

We have the result from the definition of the cup-product on $\operatorname{Hom}(W, A)$ and the fact that $\left(\left(W, \delta_{W}\right), \psi_{W}\right)$ is a differential graded coalgebra.

Let us verify that $\phi_{A}\left(u^{l+1} \otimes B\left(f_{0}\left[f 1\left|f_{2} \cdots\right| f_{k-1} \mid f_{k}\right]\right)\right)=(I d \otimes B) \circ \phi_{A}\left(u^{l+1} \otimes\right.$ $\left.f_{0}\left[f 1\left|f_{2}\right| \cdots\left|f_{k-1}\right| f_{k}\right]\right)$.

Since

$$
B\left(f_{0}\left[f 1\left|f_{2}\right| \cdots\left|f_{k-1}\right| f_{k}\right]\right)=\sum_{i=0}^{k}(-1)^{\varepsilon_{i}} 1\left[f_{i}\left|f_{i+1}\right| \cdots\left|f_{k}\right| f_{1}|\cdots| f_{i-2} \mid f_{i-1}\right]
$$

with

$$
\varepsilon_{i}=\left(\sum_{j=0}^{i-1}\left|f_{j}\right|+1\right)\left(\sum_{j=i}^{k-i+1}\left|f_{j}\right|+k-i+1\right)
$$


then

$$
\begin{aligned}
& \phi_{A}\left(u^{l+1} \otimes B\left(f_{0}\left[f 1\left|f_{2}\right| \cdots\left|f_{k-1}\right| f_{k}\right]\right)\right) \\
& =\phi_{A}\left(u^{l+1} \otimes \sum_{i=0}^{k}(-1)^{\varepsilon_{i}} 1\left[f_{i}\left|f_{i+1}\right| \cdots\left|f_{k}\right| f_{1}|\cdots| f_{i-2} \mid f_{i-1}\right]\right) \\
& =\phi_{A}\left(\sum_{i=0}^{k}(-1)^{\varepsilon_{i}} u^{l+1} \otimes 1\left[f_{i}\left|f_{i+1}\right| \cdots\left|f_{k}\right| f_{0}|\cdots| f_{i-2} \mid f_{i-1}\right]\right) \\
& =\sum_{i=0}^{k}(-1)^{\varepsilon_{i}} \phi_{A}\left(u^{l+1} \otimes 1\left[f_{i}|\cdots| f_{k}\left|f_{0}\right| \cdots \mid f_{i-1}\right]\right) \\
& =\sum_{i=0}^{k}(-1)^{\varepsilon_{i}}\left(I d \otimes I d \otimes s^{\otimes(k+1)}\right) \circ \\
& {\left[g\left(u^{l+1}\right) \otimes 1 \otimes f_{i} \otimes f_{i+1} \otimes \cdots \otimes f_{k} \otimes f_{0} \otimes \cdots \otimes f_{i-1}\right] \circ \Psi_{W}^{(k+2)}} \\
& =\sum_{i=0}^{k}(-1)^{\varepsilon_{i}} g\left(u^{l+1}\right) \otimes\left(I d \otimes s^{\otimes(k+1)}\right) \circ \\
& {\left[1 \otimes f_{i} \otimes f_{i+1} \otimes \cdots \otimes f_{k} \otimes f_{0} \cdots \otimes f_{i-1}\right] \circ \Psi_{W}^{(k+1)}} \\
& =\left(g\left(u^{l+1}\right) \otimes \sum_{i=0}^{k}(-1)^{\varepsilon_{i}}\left(I d \otimes s^{\otimes(k+1)}\right) \circ\right. \\
& \left.\left[1 \otimes f_{i} \otimes f_{i+1} \otimes f_{i+2} \otimes \cdots \otimes f_{k} \otimes f_{0} \cdots \otimes f_{i-2} \otimes f_{i-1}\right]\right) \circ \Psi_{W}^{(k+2)} \\
& =g\left(u^{l+1}\right) \otimes B\left(\left(I d \otimes s^{\otimes k}\right) \circ\left[f_{0} \otimes f_{1} \otimes f_{2} \otimes \cdots \otimes f_{k}\right]\right) \circ \psi_{W}^{(k+2)} \\
& =(I d \otimes B) \circ\left(g\left(u^{l+1}\right) \otimes\left(I d \otimes s^{\otimes k}\right) \otimes\left[f_{0} \otimes f_{1} \otimes \cdots \otimes f_{k}\right]\right) \circ \Psi_{W}^{(k+1)} \\
& =(I d \otimes B) \circ \phi_{A}\left(u^{l+1} \otimes f_{0}\left[f_{1}|\cdots| f_{k-1} \mid f_{k}\right]\right) \text {. }
\end{aligned}
$$

From the above calculations, we conclude that $\phi_{A}$ is a chain complex homomorphism such that the diagram above commutes.

Lemma 3.3. If $\left(A, d_{A}, \mu_{A}, \tilde{\kappa}_{A}\right)$ is a $\pi$-shc-algebra, then

(i) $\tilde{\kappa}_{A}$ is a strict homomorphism of shc-algebras; and

(ii) $H_{*}\left(\phi_{A}\right): H C_{*}^{-} \operatorname{Hom}(W, A) \longrightarrow H_{*}\left(\operatorname{Hom}\left(W, C^{-} A\right)\right)$ preserves the natural products.

Proof. (i) was proved in [21, A.5]. 
In order to establish (ii), consider the following commutative diagrams A and B:
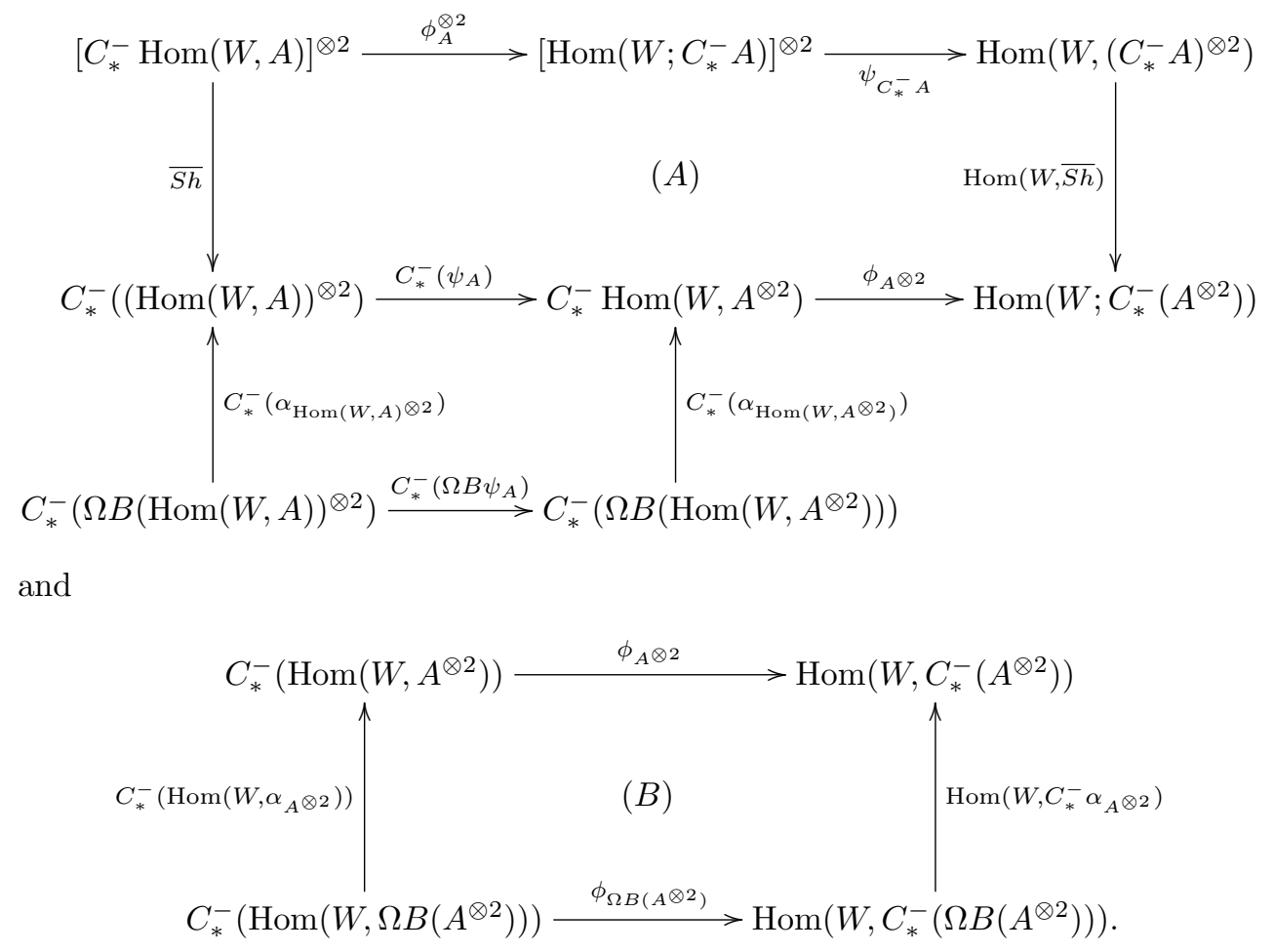

From $F_{3}, F_{8}$ and $[\mathbf{2 1}$, Lemma A.4(b)], we deduce that there exist homomorphisms of differential graded algebras

1. $\Omega B(\operatorname{Hom}(W, A)) \stackrel{\theta_{A}^{\prime}}{\longrightarrow} \operatorname{Hom}(W, \Omega B A)$

2. $\left.\operatorname{Hom}\left(W, A^{\otimes 2}\right)\right) \stackrel{\theta_{A}^{\prime} \otimes 2}{\longrightarrow} \operatorname{Hom}\left(W, \Omega B A^{\otimes 2}\right)$

3. $\Omega B\left(\operatorname{Hom}\left(W, A^{\otimes 2}\right)\right) \stackrel{\theta_{A \otimes 2}^{\prime \prime}}{\longrightarrow} \Omega B \operatorname{Hom}\left(W, \Omega B A^{\otimes 2}\right)$

such that

$$
\begin{gathered}
\operatorname{Hom}\left(W, \alpha_{A}\right) \circ \theta_{A}^{\prime}=\alpha_{\operatorname{Hom}(W, A),} \\
\operatorname{Hom}\left(W, \alpha_{A^{\otimes 2}}\right) \circ \alpha_{\operatorname{Hom}\left(W, \Omega B\left(A^{\otimes 2}\right)\right)} \circ \theta_{A^{\otimes 2}}^{\prime \prime}=\alpha_{\operatorname{Hom}\left(W, A^{\otimes 2}\right)}
\end{gathered}
$$

and

$$
\operatorname{Hom}\left(W, \alpha_{A^{\otimes 2}}\right) \circ \theta_{A^{\otimes 2}}^{\prime}=\alpha_{\operatorname{Hom}\left(W, A^{\otimes 2}\right)},
$$

since $\mu_{\operatorname{Hom}(W, A)}=\Omega B \operatorname{Hom}\left(W, \alpha \circ \mu_{A}\right) \circ \theta_{\operatorname{Hom}\left(W, A^{\otimes 2}\right)} \circ \Omega B \psi_{A}$, where $\theta_{\operatorname{Hom}\left(W, A^{\otimes 2}\right)}$ denotes the unique homomorphism of differential graded algebras such that

$$
\operatorname{Hom}\left(W, \alpha_{A \otimes 2}\right) \circ \alpha_{\operatorname{Hom}\left(W, \Omega B\left(A^{\otimes 2}\right)\right)} \circ \theta_{\operatorname{Hom}(W, A \otimes 2}=\alpha_{\operatorname{Hom}(W, A \otimes A)} .
$$


Then we obtain the following commutative diagrams C and D:

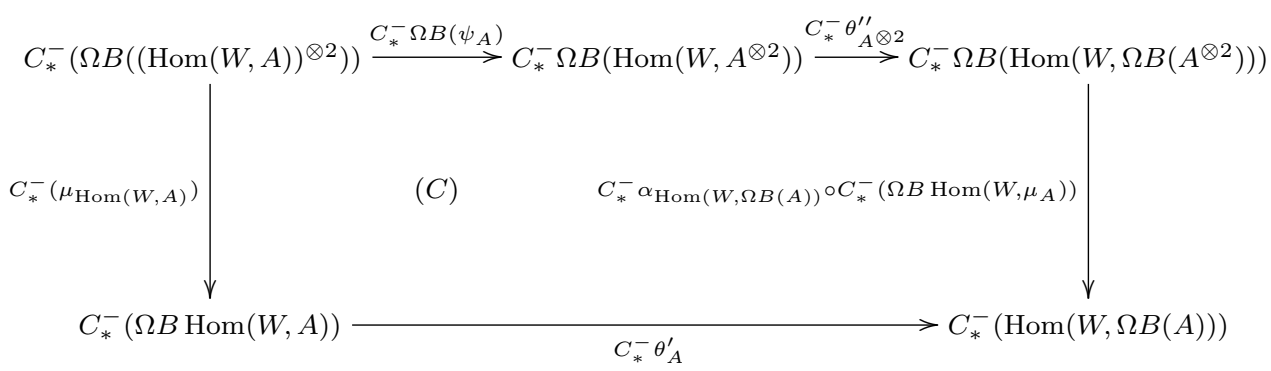

and

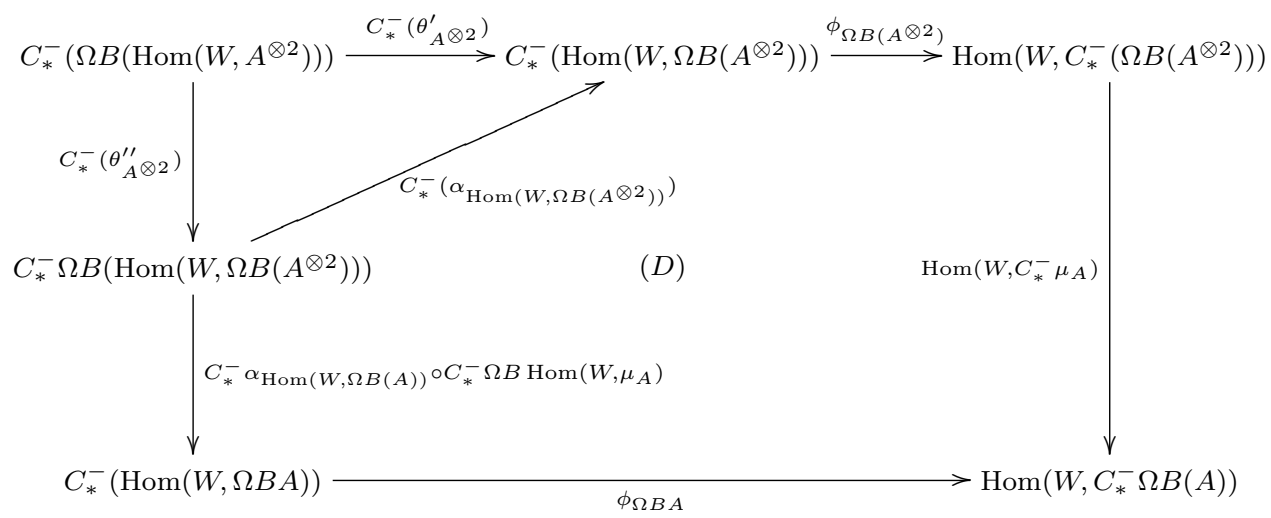

By choosing linear sections of $C^{-} \alpha_{\left(\operatorname{Hom}\left(W, A^{\otimes 2}\right)\right.}\left(\operatorname{resp} . \operatorname{Hom}\left(W, C_{*}^{-} \alpha_{A_{\otimes 2}}\right)\right)$, one can define the product $m_{C_{*}^{-}(\operatorname{Hom}(W, A))}$ on $C_{*}^{-}(\operatorname{Hom}(W, A))$ as in 2.3 (resp. $m_{\operatorname{Hom}\left(W, C_{*}^{-} A\right)}$ on $\left.\operatorname{Hom}\left(W, C_{*}^{-} A\right)\right)$. Thus by gluing together diagrams $\mathrm{A}, \mathrm{B}, \mathrm{C}$ and $\mathrm{D}$, we have the following commutative diagram:

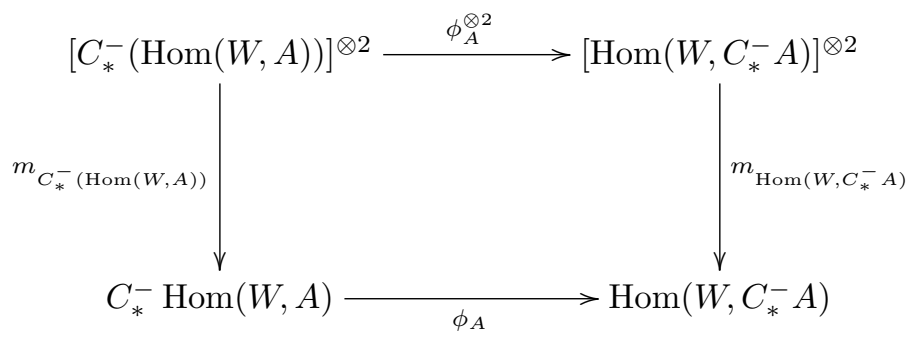

which commutes up to linear homotopy. This proves that $H_{*} \phi_{A}$ preserves the natural products.

\subsection{End of the proof of the first part of Theorem 1.1}

Let $\left(\left(A, d_{A}\right), \mu, \tilde{\kappa}_{A}\right)$ be a $\pi$-shc algebra. Since for any $p \geqslant 2, \Omega B\left(A^{\otimes p}\right)$ is a $s h c$ algebra with $\alpha_{A} \circ \mu_{A}^{(p)} \circ i_{A \otimes p}=m_{A}^{(p)}$ (see 2.1.7), hence we deduce from the definition of the $\pi$-shc algebra that the following diagram commutes up to homotopy in the 
category $\pi$-DM:

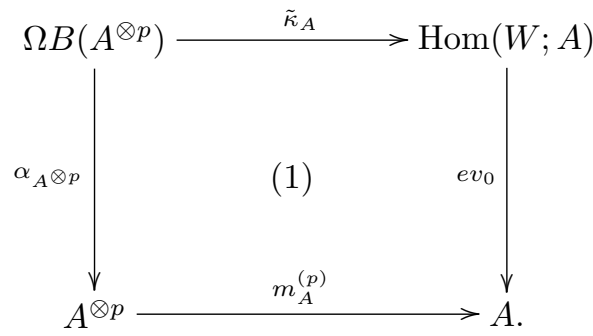

Let $S_{p}$ be the symmetric group on $\{1,2, \ldots, p\}$ and consider the action of $S_{p}$ on $C_{*}^{-}\left(A^{\otimes p}\right)$ (resp. on $C_{*}^{-}\left(\Omega B\left(A^{\otimes p}\right)\right)$ ) defined by the following rule: $\sigma\left(u^{l} \otimes b_{0}\left[b_{1}\left|b_{2}\right| \cdots\right.\right.$ $\left.\left.\left|b_{s-1}\right| b_{s}\right]\right)=u^{l} \otimes \sigma b_{0}\left[\sigma b_{1}\left|\sigma b_{2}\right| \cdots\left|\sigma b_{s-1}\right| \sigma b_{s}\right], b_{i} \in A^{\otimes p} \quad\left(\right.$ resp. $b_{i} \in \Omega B\left(A^{\otimes p}\right)$ ) so that $C_{*}^{-} A^{\otimes p}, C_{*}^{-} \Omega B\left(A^{\otimes p}\right)$ are $\pi$-chain complexes and $\Omega B\left(A^{\otimes p}\right) \stackrel{\alpha_{A}^{\otimes p}}{\rightarrow} A^{\otimes p}, C_{*}^{-}\left(\Omega B\left(A^{\otimes p}\right)\right)$ $C_{*}^{-} \stackrel{\left(\alpha_{A} \otimes p\right)}{\longrightarrow} C_{*}^{-}\left(A^{\otimes p}\right)$ are quasi-isomorphisms of $\pi$-chain complexes.

Consider on the other hand the $\pi$-chain complex homomorphism $\left[C_{*}^{-} A\right]^{\otimes p} \stackrel{\overline{S h}(p)}{\rightarrow}$ $C_{*}^{-}\left(A^{\otimes p}\right)$, called a $p$-iterated cyclic shuffle map and defined by induction as follows: $\overline{S h}=S h \circ\left(m_{\mathbb{K}[u]} \otimes I d\right) \circ(i d \otimes T \otimes i d), \overline{S h}{ }^{(2)}=\overline{S h} \circ(\overline{S h} \otimes i d)$ and for all $p \geqslant$ $2, \overline{S h}{ }^{(p)}=\overline{S h}^{(p)}\left(\overline{S h}^{(p-1)} \otimes i d\right)\left(\right.$ where $S h=(i d \otimes s h)+u\left(i d \otimes s h^{\prime}\right)$ denotes the cyclic shuffle map). Indeed we have for any

$$
\begin{gathered}
x=x_{n_{1}} \otimes x_{n_{2}} \otimes \cdots \otimes x_{n_{p-1}} \otimes x_{n_{p}} \in\left[C_{*}^{-} A\right]^{\otimes p}, \\
\left(x_{n_{i}}=u^{l_{n_{i}}} \otimes a_{0}^{n_{i}}\left[a_{1}^{n_{i}}\left|a_{2}^{n_{i}}\right| \cdots\left|a_{s_{i}-1}^{n_{i}}\right| a_{s_{i}}^{n_{i}}\right] \in C_{*}^{-} A\right)_{i=1}^{p} \\
\overline{S h}{ }^{(p)}(x)=u^{l_{n_{1}}+\cdots+l_{n_{p}} \otimes\left(a_{0}^{n_{1}} \otimes \cdots \otimes a_{0}^{n_{p}}\right)} \\
\otimes \sum_{\sigma}(-1)^{\epsilon_{\sigma}}\left[a_{1}^{n_{1}} \otimes 1 \otimes \cdots \otimes 1|\cdots| a_{s_{1}}^{n_{1}} \otimes 1 \otimes \cdots \otimes 1\left|1 \otimes a_{1}^{n_{2}} \otimes 1 \otimes \cdots \otimes 1\right| \cdots\right. \\
\left.\left|1 \otimes a_{s_{2}}^{n_{2}} \otimes 1 \otimes \cdots \otimes 1\right| \cdots\left|1 \otimes 1 \otimes \cdots \otimes 1 \otimes a_{1}^{n_{p}}\right| \cdots \mid 1 \otimes 1 \otimes \cdots \otimes 1 \otimes a_{s_{p}}^{n_{p}}\right] \\
+u^{l_{n_{1}}+\cdots+l_{n_{p}}+p} \otimes 1 \otimes 1 \otimes \cdots \otimes 1 \\
\otimes \sum_{\sigma^{\prime}}(-1)^{\epsilon_{\sigma^{\prime}}}\left[a_{0}^{n_{1}} \otimes 1 \otimes \cdots \otimes 1|\cdots| a_{0}^{n_{p}} \otimes 1 \otimes \cdots \otimes 1\left|1 \otimes a_{1}^{n_{1}} \otimes 1 \otimes \cdots \otimes 1\right| \cdots\right. \\
\left|1 \otimes a_{s_{1}}^{n_{1}} \otimes 1 \otimes \cdots \otimes 1\right| 1 \otimes 1 \otimes a_{1}^{n_{2}} \otimes 1 \otimes \cdots \otimes 1 \mid \cdots \\
\left.\left|1 \otimes a_{s_{2}}^{n_{2}} \otimes 1 \otimes \cdots \otimes 1\right| \cdots \mid 1 \otimes 1 \otimes \cdots \otimes 1 \otimes a_{s_{p}}^{n_{p}}\right]
\end{gathered}
$$

with $\sigma$ a $\left(n_{1}, n_{2}, \ldots, n_{p}\right)$-shuffle and $\sigma^{\prime}$ a $\left(n_{1}, n_{2}, \ldots, n_{p}\right)$-cyclic shuffle. Since for every $\sigma \in S_{p}, x \in\left(C_{*}^{-}(A)\right)^{\otimes p}, \sigma x=x_{\sigma\left(n_{1}\right)} \otimes x_{\sigma\left(n_{2}\right)} \otimes \cdots \otimes x_{\sigma\left({ }_{n} p\right)}$, the sets of $\left(n_{1}, n_{2}\right.$, $\left.\ldots, n_{p}\right)$-shuffles and $\left(n_{1}, n_{2}, \cdots n_{p}\right)$-cyclic shuffles coincide respectively with the set of $\left(\sigma\left(n_{1}\right), \sigma\left(n_{2}\right), \ldots, \sigma\left({ }_{n} p\right)\right)$-shuffles and the set of $\left(\sigma\left(n_{1}\right), \sigma\left(n_{2}\right), \ldots, \sigma\left({ }_{n} p\right)\right)$-cyclic shuffles, then the $p$-iterated cyclic shuffle map $\overline{S h}{ }^{(p)}$ is $\pi$ linear and we obtain the following sequence of $\pi$-quasi-isomorphisms:

$$
C_{*}^{-}\left(\Omega B\left(A^{\otimes p}\right)\right) \stackrel{C_{*}^{-}\left(\alpha_{A} \otimes p\right)}{\longrightarrow} C_{*}^{-}\left(A^{\otimes p}\right) \stackrel{\overline{S h}^{(p)}}{\longleftarrow}\left[C_{*}^{-} A\right]^{\otimes p} .
$$


Let $\phi_{A}$ be the map defined in the proof of Proposition 3.2. By applying the functor $C_{*}^{-}(-)$to the diagram (1) above and using Lemma 2.2 and the definition of the product $m_{C_{*}^{-} A}^{(p)}$ on $C_{*}^{-} A$, we obtain the following the diagram commutative up to homotopy;

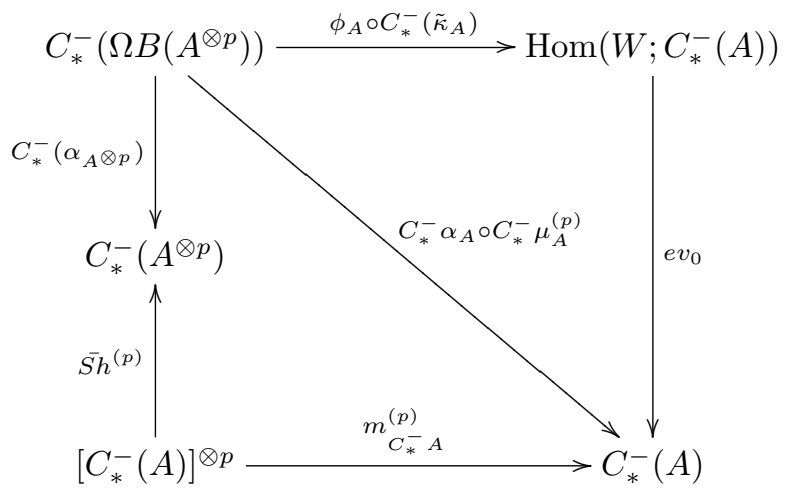

which induces the following commutative diagram in homology:

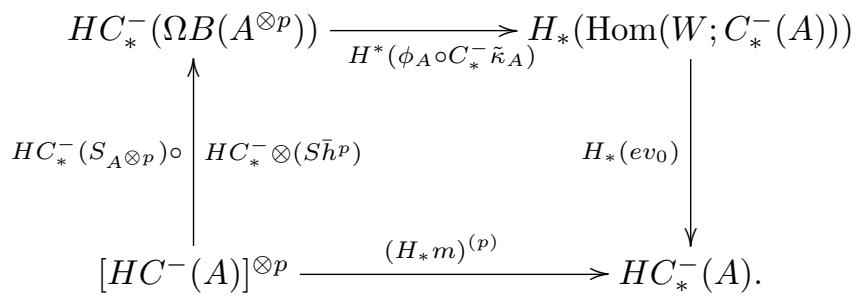

Thus $C_{*}^{-}(A)$ together with the structural map $\theta=\phi_{A} \circ C_{*}^{-}\left(\tilde{\kappa}_{A}\right)$ is a Dold quasialgebra. From the lines of the proof of May[15, Proposition 2.3], this diagram defined algebraic Steenrod operations on $H C^{-}(A)$.

Following the same lines, Proposition 2.1 can still be used to prove the Cartan formulas $[\mathbf{2 1}]$.

\section{Proof of the second part of Theorem 1.1}

Let us begin this section by giving the proof of the following result due to Bitjong Ndombol and Jean-Claude Thomas [21, Theorem B].

Proposition 4.1. Let $X$ be a topological space. The algebra $N^{*}(X)$ is a natural $\pi$ shc-algebra.

Proof. Here we replace the ground field $\mathbb{F}_{p}$ by $\mathbb{F}_{p}[\pi]$.

Munkholm established in $[\mathbf{1 7}, 4-7]$ that there exists a natural transformation $\mu_{X}: \Omega B\left(N^{*}(X) \otimes N^{*}(X)\right) \longrightarrow \Omega B\left(N^{*}(X)\right.$ such that $\alpha_{N^{*}(X)} \circ \mu_{X} \circ i_{N^{*}(X) \otimes N^{*}(X)}=$ $m_{N^{*}(X)}$, where $m_{N^{*}(X)}$ denotes the usual product on $N^{*}(X)$. Furthermore $\left(N^{*}(X)\right.$, $\left.\mu_{X}\right)$ is a shc-algebra. 
Let $\Delta$ denote the topological diagonal. From [21, A.2], $F_{3}$, and the fact that the Eilenberg-Zilber map $E Z$ is a trivialized extension, we obtain the following commutative diagram:

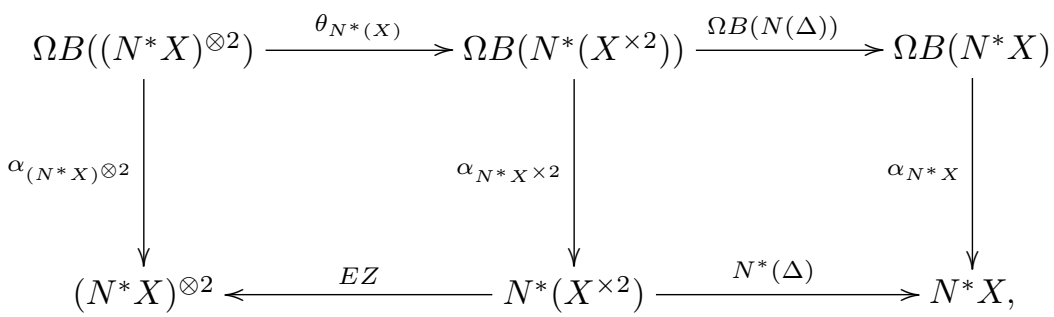

from which we define the following homomorphism of differential graded algebras

$$
\tilde{\mu}_{X}=\Omega B(N(\Delta)) \circ \theta_{N^{*}(X)} .
$$

Moreover there exists a homomorphism of differential graded algebras

$$
\Omega B\left(N^{*} X\right) \stackrel{\tilde{\theta}_{N^{*} X}}{\longrightarrow} \operatorname{Hom}\left(W, N^{*} X\right)
$$

deduced from $\left[\mathbf{2 1}\right.$, Lemma A.4(a)] and $F_{3}$, which rise to the commutative diagram

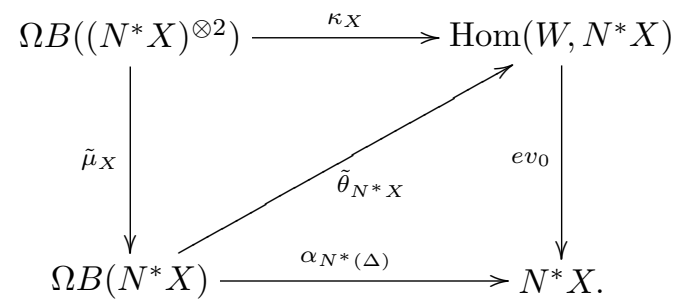

Thus we obtain a $\mathbb{F}_{p}[\pi]$-homomorphism of differential graded algebras $\kappa_{X}$ defined by $\kappa_{X}=\theta_{N^{*} X} \circ \tilde{\mu}_{X}$ such that $e v_{0} \circ \kappa_{X}=\alpha_{N^{*}(\Delta)} \circ \tilde{\mu}_{X}$. To end this proof it is enough to establish that $\tilde{\mu}_{X} \simeq_{D A} \mu_{X}$. We remark that $\tilde{\mu}_{X} \simeq_{D A} \mu_{X}$ if and only if $t_{X} \simeq_{T} \tilde{t}_{X}$ where $t_{X}, \tilde{t}_{X} \in T\left(B\left(N^{*}(X)\right)^{\otimes 2}, \Omega B\left(N^{*}(X)\right)\right)$ denote the universal twisting cochains associated respectively to $\mu_{X}$ and $\tilde{\mu}_{X}[\mathbf{1 7}]$. Finally, it is necessary to find $H \in$ $\operatorname{Hom}^{1}\left(\Omega B\left(\left(N^{*} X\right)^{\otimes 2}\right), \Omega B N^{*} X\right)$ such that $H: t_{X} \simeq_{T} \tilde{t}_{X}$. i.e., $D H=\tilde{t}_{X} \cup H-H \cup$ $t_{X}$ and $H \circ \eta_{B N^{*} X \otimes p}=\eta_{B N^{*} X} ; \varepsilon_{\Omega B N^{*} X} \circ H=\varepsilon_{B N^{*} X}$. We define $H$ by the following induction formula:

$$
\begin{aligned}
H & =h\left(\tilde{t}_{X} \cup H-H \cup t_{X}\right)+\eta_{\Omega B N^{*} X} \circ \varepsilon_{B N^{*} X^{\otimes p}} \\
H \circ i_{0} & =H \circ \eta_{B N^{*} X \otimes p}=\eta_{\Omega B N^{*} X},
\end{aligned}
$$

where $h: 1_{\Omega B N^{*} X} \simeq i_{N^{*} X} \circ \alpha_{N^{*} X}$ and $i_{0}=\eta_{B\left(N^{*} X\right) \otimes p}$. Since

$$
\tilde{t}_{X} \cup H=m_{\Omega B\left(N^{*} X\right)} \circ\left(\tilde{t}_{X} \otimes H\right) \circ \Delta_{B\left(N^{*} X\right) \otimes p}
$$

and

$$
H \cup t_{X}=m_{\Omega B\left(N^{*} X\right)} \circ\left(H \otimes t_{X}\right) \circ \Delta_{B\left(N^{*} X\right) \otimes p},
$$

then

$$
H=h \circ m_{\Omega B\left(N^{*} X\right)} \circ\left(\tilde{t}_{X} \otimes H-H \otimes t_{X}\right) \circ \Delta_{B\left(N^{*} X\right)^{\otimes p}}+\eta_{\Omega B\left(N^{*} X\right)} \circ \varepsilon_{\Omega B\left(N^{*} X\right)^{\otimes p}} .
$$


Notice that

$$
\begin{gathered}
\varepsilon_{B N^{*} X \otimes p} \circ \eta_{B\left(N^{*} X\right)^{\otimes p}}=I d_{\mathbb{K}} ; \\
\Delta_{B\left(N^{*} X\right)^{\otimes p}} \circ \eta_{B\left(N^{*} X\right)^{\otimes p}}=\eta_{B\left(N^{*} X\right)^{\otimes p}} \otimes \eta_{B\left(N^{*} X\right)^{\otimes p}}
\end{gathered}
$$

and

$$
\tilde{t}_{X} \circ \eta_{B\left(N^{*} X\right)^{\otimes p}}=t_{X} \circ \eta_{B\left(N^{*} X\right) \otimes p}=0
$$

so

$$
\begin{aligned}
H \circ i_{0} & =H \circ \eta_{B\left(N^{*} X\right)^{\otimes p}}=h \circ m_{\Omega B\left(N^{*} X\right)} \circ\left(\tilde{t}_{X} \circ \eta_{B\left(N^{*} X\right)^{\otimes p}} \otimes H \eta_{B\left(N^{*} X\right)^{\otimes p}}\right. \\
& \left.-H \eta_{B\left(N^{*} X\right)^{\otimes p}} \otimes t_{X} \circ \eta_{B\left(N^{*} X\right)^{\otimes p}}\right)+\eta_{\Omega B\left(N^{*} X\right)}=\eta_{\Omega B\left(N^{*} X\right)} .
\end{aligned}
$$

More generally, suppose that $H \circ i_{j}$ can be written as (I) for $j \leqslant k$ and let us prove that $H \circ i_{k+1}$ can be written by formula (I) and $H \circ i_{j}\left(j \leqslant k<k^{\prime}\right)$.

$$
\begin{aligned}
H \circ i_{k^{\prime}}= & h \circ m_{\Omega B\left(N^{*} X\right)}\left(\tilde{t}_{X} \otimes H-H \otimes t_{X}\right) \circ \Delta_{B\left(N^{*} X\right) \otimes p} \circ i_{k^{\prime}} \\
& +\eta_{\Omega B\left(N^{*} X\right)} \circ \varepsilon_{\Omega B\left(N^{*} X\right)^{\otimes p}} \circ i_{k^{\prime}} \\
= & h \circ m_{\Omega B\left(N^{*} X\right)} \circ\left[\tilde{t}_{X} \otimes H-H \otimes t_{X}\right]\left[\eta_{B\left(N^{*} X\right)^{\otimes p}} \otimes i_{k^{\prime}}\right. \\
+ & \left.\sum_{\nu=1}^{k^{\prime}} i_{\nu} \otimes i_{k^{\prime}-\nu}+i_{k^{\prime}} \otimes \eta_{B\left(N^{*} X\right)^{\otimes p}}\right]+\eta_{\Omega B\left(N^{*} X\right)} \circ \varepsilon_{\Omega B\left(N^{*} X\right)^{\otimes p}} \circ i_{k^{\prime}}
\end{aligned}
$$

where

$$
\begin{aligned}
(s(I A))^{\otimes \nu} & \stackrel{i_{\nu}}{\longrightarrow} B A \\
\left(s\left(a_{1}\right) \otimes s\left(a_{2}\right) \otimes \cdots \otimes s\left(a_{\nu}\right)\right) & \longmapsto i_{\nu}\left(s\left(a_{1}\right) \otimes s\left(a_{2}\right) \otimes \cdots \otimes s\left(a_{\nu}\right)\right)=\left[a_{1}|\cdots| a_{\nu}\right]
\end{aligned}
$$$$
\left(A=\left(N^{*} X\right)^{\otimes p}\right) \text {. In particular } i_{k \prime}(s(a))=[a] \text { and }
$$$$
\varepsilon_{N^{*} X}\left(\left[a_{1}|\cdots| a_{k}\right]\right)= \begin{cases}0 & \text { if } k>0 \\ 1 & \text { if } k=0\end{cases}
$$

and

$$
\varepsilon_{N^{*} X \otimes p} \circ i_{k}\left(s a_{1} \otimes s a_{2} \otimes \cdots \otimes s a_{k}\right)= \begin{cases}0 & \text { if } k>0 \\ 1 & \text { if } k=0\end{cases}
$$

$\Delta_{B\left(N^{*} X\right)} \circ i_{k^{\prime}}=\eta_{B\left(N^{*} X\right) \otimes p} \otimes i_{k^{\prime}}+\sum_{\nu=1}^{k^{\prime}} i_{\nu} \otimes i_{k^{\prime}-\nu}+i_{k^{\prime}} \otimes \eta_{B\left(N^{*} X\right)^{\otimes p}}$.

Thus we deduce that

$$
\begin{aligned}
H \circ i_{k^{\prime}} & =h \circ m_{\Omega B\left(N^{*} X\right)}\left(\tilde{t}_{X} \otimes H-H \otimes t_{X}\right)\left(i_{0} \otimes i_{k^{\prime}}+\sum_{\nu=1}^{k^{\prime}-1} i_{\nu} \otimes i_{k^{\prime}-\nu}+i_{k^{\prime}} \otimes i_{0}\right) \\
& =h \circ m_{\Omega B\left(N^{*} X\right)}\left[\sum_{\nu=0}^{k \prime}\left(\tilde{t}_{X} \circ i_{\nu}\right) \otimes\left(H \circ i_{k^{\prime}-\nu}\right)-\sum_{\nu=0}^{k \prime} H \circ i_{\nu} \otimes t_{X} \circ i_{k^{\prime}-\nu}\right] \\
& =h \circ m_{\Omega B\left(N^{*} X\right)}\left[\sum_{\nu=1}^{k^{\prime}}\left(\tilde{t}_{X} \circ i_{\nu}\right) \otimes\left(H \circ i_{k^{\prime}-\nu}\right)-\sum_{\nu=0}^{k^{\prime}-1}\left(H \circ i_{\nu}\right) \otimes\left(t_{X} \circ i_{k^{\prime}-\nu}\right)\right] .
\end{aligned}
$$

Let us verify that $D H=\tilde{t}_{X} \cup H-H \cup t_{X}$. 
Since $D H=D\left[h\left(\tilde{t}_{X} \cup H-H \cup t_{X}\right)\right]+D\left(\eta_{\Omega B\left(N^{*} X\right)} \circ \varepsilon_{\Omega B\left(N^{*} X\right)^{\otimes p}}\right.$ with

$$
\begin{aligned}
D\left(\eta_{\Omega B\left(N^{*} X\right)} \circ \varepsilon_{\Omega B\left(N^{*} X\right)^{\otimes p}}\right) & =\underbrace{d_{\Omega B\left(N^{*} X\right)} \eta_{\Omega B\left(N^{*} X\right)}}_{0} \varepsilon_{\Omega B\left(N^{*} X\right)^{\otimes p}} \\
& -\eta_{\Omega B\left(N^{*} X\right)} \underbrace{\varepsilon_{\Omega B\left(N^{*} X\right) \otimes p} d_{\Omega B\left(N^{*} X\right)^{\otimes p}}}_{0}, \\
& =0
\end{aligned}
$$

we also have $D h=1_{\Omega B\left(N^{*} X\right)}-i_{N^{*} X} \circ \alpha_{N^{*} X}$ and $D f=d f-(-1)^{|f|} f d$.

Thus

$$
\begin{aligned}
D H= & (D h)\left[\tilde{t}_{X} \cup H-H \cup t_{X}\right]+h\left(D\left[\tilde{t}_{X} \cup H-H \cup t_{X}\right]\right)= \\
& \left(1_{\Omega B\left(N^{*} X\right)}-i_{N^{*} X} \circ \alpha_{N^{*} X}\right)\left[\tilde{t}_{X} \cup H-H \cup t_{X}\right]+h\left(D\left[\tilde{t}_{X} \cup H-H \cup t_{X}\right]\right) .
\end{aligned}
$$

Since $\alpha_{N^{*} X} \circ h=0$ and $D\left[\tilde{t}_{X} \cup H-H \cup t_{X}\right]=0$, we deduce that $D H=\tilde{t}_{X} \cup H-$ $H \cup t_{X}$, hence $\tilde{t}_{X} \simeq_{T} t_{X}$.

\subsection{End of the proof of the second part of the theorem}

Consider the simplicial model $K$ of the unit cycle $S^{1}$ and the cosimplicial model space $\underline{X}$ defined by $\underline{X}=\operatorname{Map}(K(n), X)$ whose geometric resolution $\|\underline{X}\|$ is homeomorphic to $L X,[\mathbf{2 0}$, Part II-3]. In [10, Lemma 5.5, Proof of Theorem A and Theorem B], J.D.S. Jones has constructed the $\mathbb{F}_{p}[u]$-modules quasi-isomorphism $C_{*}^{-} N^{*} X \stackrel{\Psi}{\longrightarrow} \mathbb{F}_{p}[u] \otimes N^{*}(\|X\|)$ which induces a graded algebra isomorphism $H C_{*}^{-} X \cong H_{S^{1}}^{-*}\left(L X, \mathbb{F}_{p}\right),[\mathbf{1 8}]$. Following the lines of $[\mathbf{2 1}]$, consider a $\pi$-shc differential graded algebra $\left(N^{*} X, \mu_{X}, \tilde{\kappa}_{X}\right)$ endowed with the structural map $\hat{\theta}_{X}=\phi_{N^{*} X} \circ C^{-} \tilde{\kappa}_{X}$ defining the algebraic Steenrod operations on $H C_{*}^{-}\left(N^{*} X\right)$ and $W \otimes\left(N^{*}(\|\underline{X}\|)\right)^{\otimes p} \stackrel{\Gamma_{X}}{\longrightarrow} N^{*}(\|\underline{X}\|)$ the structural map defining Steenrod operations on $\left.N^{*}(\|\underline{X}\|)\right)$, [15, 7.5]. Define the map $\gamma_{X}$ as the composite

$$
\begin{gathered}
W \otimes\left(\mathbb{F}_{p}[u] \otimes N^{*}(\|\underline{X}\|)\right)^{\otimes p} \stackrel{i d \otimes(i d \otimes T \otimes i d)^{\otimes p}}{\longrightarrow} \\
W \otimes\left(\mathbb{F}_{p}[u]\right)^{\otimes p} \otimes\left(N^{*}(\|\underline{X}\|)\right)^{\otimes p} \stackrel{i d \otimes m_{\mathbb{F}_{p}[u]}^{(p)} \otimes i d}{\longrightarrow} \\
W \otimes\left(\mathbb{F}_{p}[u]\right) \otimes\left(N^{*}(\|\underline{X}\|)\right)^{\otimes p} \stackrel{T \otimes i d}{\longrightarrow}\left(\mathbb{F}_{p}[u]\right) \otimes W \otimes\left(N^{*}(\|\underline{X}\|)\right)^{\otimes p} \stackrel{i d \otimes \Gamma_{X}}{\longrightarrow} \\
\mathbb{F}_{p}[u] \otimes N^{*}(\|\underline{X}\|) ;
\end{gathered}
$$

$\gamma_{X}$ is the structural map defining Steenrod operations on $\left(\mathbb{F}_{p}[u] \otimes N^{*}(\|\underline{X}\|)\right)$ and inducing the chain map $\tilde{\gamma}_{X}$ (see Proposition 2.1). Consider the following diagram:

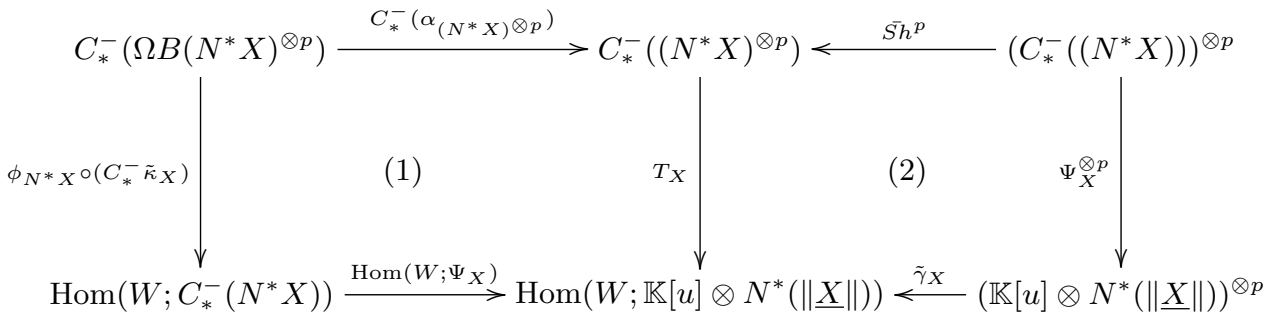


where the functors $X \longrightarrow C_{*}^{-}\left(\left(N^{*} X\right)^{\otimes p}\right)$ and $X \longrightarrow \operatorname{Hom}\left(W ; \mathbb{K}[u] \otimes N^{*}(\|\underline{X}\|)\right)$ defined on the category Top with models $\mathcal{M}=\left\{\mathbb{O}^{n}, \mathbb{O}^{n}=\bigvee_{p \geqslant 0}\left(\bigvee^{p+1}\left(\triangle^{n} \times \triangle^{p}\right)\right) ; n \in\right.$ $\mathbb{N}\}$ preserve the units and are respectively acyclic and corepresentable. We obtain from the equivariant cyclic model theorem (see [21, Appendix B] and [18]) that there exists a $\pi$-linear natural transformation $C_{*}^{-}\left(\left(N^{*} X\right)^{\otimes p}\right) \stackrel{T_{X}}{\longrightarrow} \operatorname{Hom}(W ; \mathbb{K}[u] \otimes$ $\left.N^{*}(\|\underline{X}\|)\right)$ such that $T \circ C_{*}^{-}\left(\alpha_{\left(N^{*} X\right) \otimes p}\right) \simeq_{\pi} \operatorname{Hom}\left(W ; \Psi_{X}\right) \circ \phi_{N^{*} X} \circ\left(C_{*}^{-} \tilde{\kappa}_{X}\right)$. Consequently the Jones isomorphism respects Steenrod operations.

\section{5. $\pi$-shc models, $[21,3]$}

\subsection{Minimal algebra}

Let $V=\left\{V^{i}\right\}_{i \geqslant 1}$ be a graded vector space and let $\left(T V, d_{V}\right)$ denotes the free differential graded algebra generated by $V: T^{r} V=V \otimes V \otimes \cdots \otimes V$ ( $r$ times) and $v_{1} \cdot v_{2} \cdots v_{k} \in(T V)^{n}$ if $\sum_{i=1}^{k}\left|v_{i}\right|=n$. The differential $d_{V}$ on $T V$ is the unique degree 1 derivation on $T V$ defined by a given linear map $V \longrightarrow T V$ and such that $d_{V} \circ d_{V}=0$. The differential $d_{V}: T V \longrightarrow T V$ decomposes as $d_{V}=d_{0}+d_{1}+\cdots$ with $d_{k} V \subset T^{k+1} V$. If we assume that $V^{1}=0$ and $d_{0}=0$ then $\left(T V, d_{V}\right)$ is called a 1 connected minimal algebra. For any differential graded algebra $\left(T U, d_{U}\right)$ such that $H^{0}\left(T U, d_{U}\right)=\mathbb{F}_{p}$ and $H^{1}\left(T U, d_{U}\right)=0$, there exists a sequence of homomorphisms of differential graded algebras, $\left(T U, d_{U}\right) \stackrel{P_{V}}{\longrightarrow}\left(T V, d_{V}\right) \stackrel{\varphi_{V}}{\longrightarrow}\left(T U, d_{U}\right)$ where $\left(T V, d_{V}\right)$ denotes a 1-connected minimal algebra, $\varphi_{V} \circ P_{V} \simeq_{D A} i d$ and $P_{V} \circ \varphi_{V}=i d$ such that $V \cong H\left(U, d_{U}\right)$ (see $[\mathbf{2 0}, 3.1]$ or $\left.[\mathbf{2 1}, 6.4]\right)$. Moreover $\left(T V, d_{V}\right)$ is unique up to isomorphism.

\subsection{Minimal model of a product}

Assume that $\left(A, d_{A}\right)$ is a differential graded algebra such that $H^{0}(A)=\mathbb{F}_{p}$ and $H^{1}(A)=0$ and let $\left(T U[n], d_{U[n]}\right)=\Omega\left((B A)^{\otimes n}\right), n \geqslant 1$. Following the discussion above, we obtain a sequence

$$
\left(T U[n], d_{U[n]}\right)=\Omega\left((B A)^{\otimes n}\right) \stackrel{P_{V[n]}}{\longrightarrow}\left(T V[n], d_{V[n]}\right) \stackrel{\varphi_{V[n]}}{\longrightarrow}\left(T U[n], d_{U[n]}\right)
$$

with

$$
\begin{aligned}
V[n] & =s^{-1}\left(\overline{H\left((B A)^{\otimes n}\right)}\right) \cong s^{-1}\left(\overline{(H(B A))^{\otimes n}}\right)=s^{-1}\left(\overline{\left(\mathbb{F}_{p} \oplus s V\right)^{\otimes n}}\right) \\
& \cong\left(\bigoplus_{k=1}^{n}\left(\left(\mathbb{F}_{p}\right)^{\otimes k-1} \otimes V \otimes\left(\mathbb{F}_{p}\right)^{\otimes n-k}\right)\right) \oplus \cdots \oplus s^{-1}(s V \otimes s V \otimes \cdots \otimes s V) .
\end{aligned}
$$

For $n=1, V[1]=V=s^{-1} \overline{H(B A)}$ and the composite

$$
\psi_{V}=\alpha_{A} \circ \varphi_{V}:\left(T V, d_{V}\right) \longrightarrow A
$$

is a quasi-isomorphism. The algebra $\left(T V, d_{V}\right)$ is called a 1-connected minimal model of $A$.

For $n \geqslant 2$, consider the homomorphism $q_{\widehat{V}}:\left(T V[n], d_{V[n]}\right) \longrightarrow\left(T V, d_{V}\right)^{\otimes n}$ defined by $q_{\widehat{V}}(y)=1^{\otimes k-1} \otimes y \otimes 1^{\otimes n-k}$, if $y \in V_{k}:=\mathbb{F}_{p}^{\otimes k-1} \otimes V \otimes \mathbb{F}_{p}^{\otimes n-k}, \quad k \in\{1 ; 2 ; \ldots n\}$ 
and $q_{\widehat{V}}(y)=0$ if $y \in V[n]-\bigoplus_{i=1}^{n} V_{i}$. The composite

$$
\left(T V[n], d_{V[n]}\right) \stackrel{q_{\widehat{V}}}{\longrightarrow}\left(T V, d_{V}\right)^{\otimes n} \stackrel{\left(\psi_{V}\right)^{\otimes n}}{\longrightarrow} A^{\otimes n}
$$

is a quasi-isomorphism $([\mathbf{2 0}])$. Therefore $\left(T V[n], d_{V[n]}\right)$ is a minimal model of $A^{\otimes n}$.

\section{3. $\pi$-shc minimal models}

For any $n>1$, the cyclic group $S_{n}$ acts on $\widehat{V}=V[n] \subset s^{-1}(H(B A))^{\otimes n}$. This action extends diagonally on $T V[n]$ so that $d_{V[n]}$ and the homomorphism $\left(\psi_{V}\right)^{\otimes n} \circ q_{V[n]}$ are $S_{n}$-linear. Since $\alpha_{A \otimes n}$ is a $S_{n}$-equivariant quasi-isomorphism, we deduce from $\left[\mathbf{2 1}\right.$, Lemma 3.3] that the composite $\left(\psi_{V}\right)^{\otimes n} \circ q_{V[n]}$ lifts to a homomorphism of differential graded algebra $L:\left(T V[n] d_{V[n]}\right) \longrightarrow \Omega B\left(A^{\otimes n}\right)$ which is $S_{n}$-equivariant and $\alpha_{A \otimes n} \circ L=\left(\psi_{V}\right)^{\otimes n} \circ q_{V[n]}$.

Let $\left(\left(A, d_{A}\right), \mu_{A}\right)$ be an augmented shc-algebra and assume that $H^{0}(A)=\mathbb{F}_{p}$ and $H^{0}(A)=0$. Define the composite $\mu_{V}^{(n)}=P_{V} \circ \mu_{A}^{(n)} \circ L:\left(T V[n], d_{V[n]}\right) \longrightarrow\left(T V, d_{V}\right)$ such that $\mu_{V}^{(2)}=\mu_{V}:\left(T V[2], d_{V[2]}\right) \longrightarrow\left(T V, d_{V}\right)$. The triple $\left(\left(T V, d_{V}\right), \mu_{V}\right)$ is called a shc-minimal model for $\left(\left(A, d_{A}\right), \mu_{A}\right)[\mathbf{2 0}$, Section 6$]$.

Let $\left(\left(A, d_{A}\right), \mu_{A}, \tilde{\kappa}_{A}\right)$ be an augmented $\pi$-shc-algebra and assume that $H^{0}(A)=\mathbb{F}_{p}$ and $H^{0}(A)=0$. Following [21, Lemma 3.3], the composite $\tilde{\kappa}_{A} \circ L$ lifts to $S_{p}$-equivariant homomorphism of algebras $\hat{\kappa}_{A}:\left(T V[p], d_{V[p]}\right) \longrightarrow \operatorname{Hom}(W, \Omega B A)$. Hence the composite $\tilde{\kappa}_{V}=\operatorname{Hom}\left(W, P_{V}\right) \circ \hat{\kappa}_{A}:\left(T V[p], d_{V[p]}\right) \longrightarrow \operatorname{Hom}(W, T V)$ is a $S_{p}$-equivariant homomorphism of algebras and the triple $\left(\left(T V, d_{V}\right), \mu_{V}, \tilde{\kappa}_{V}\right)$ is called the $\pi$-shcminimal model for the $\left(\left(A, d_{A}\right), \mu_{A}, \tilde{\kappa}_{A}\right)[\mathbf{2 1}, 3.4]$.

\section{Examples}

In this section, the characteristic of the field $\mathbb{F}_{p}$ is $p=2$.

\subsection{Projective space $\mathbb{C} P^{\infty}$}

Let $X=\mathbb{C} P^{\infty}, H^{*}(X ; \mathbb{K})=\Lambda(x)=\mathbb{F}_{2}[x]$ with $|x|=2$. Thus the graded algebras $H C_{*}^{-}\left(N^{*} \mathbb{C} P^{\infty} ; \mathbb{F}_{2}\right)$ and $H C_{*}^{-}(\Lambda(x))$ are isomorphic. It also follows that the algebraic Steenrod operations on $H C_{*}^{-}\left(N^{*} \mathbb{C} P^{\infty} ; \mathbb{F}_{2}\right)$ are computed by those on $H C_{*}^{-}(\Lambda(x))$.

Since $\left((\Lambda(x), 0), \mu_{\Lambda(x)}, \tilde{\kappa}_{\Lambda(x)}\right)$ is a 1-connected $\pi$-shc differential graded algebra together with finite generated cohomology groups such that the shc structural map $\mu_{\Lambda(x)}=\Omega B\left(\left(m_{\Lambda(x)}\right)\right)$ and the $\pi$-shc structural map $\Omega B\left(\Lambda(x)^{\otimes 2}\right) \stackrel{\tilde{\kappa}_{\Lambda(x)}}{\rightarrow} \operatorname{Hom}(W ; \Lambda(x))$ defined on the generic elements as follows:

$$
\text { For any } \begin{aligned}
y= & <c_{1}\left|c_{2}\right| \cdots\left|c_{r-1}\right| c_{r}>, \\
c_{i} & =\left[b_{1}^{i}\left|b_{2}^{i}\right| \cdots\left|b_{l_{i}-1}^{i}\right| b_{l_{i}}^{i}\right], \\
b_{j}^{i} & =\underbrace{(x \wedge x \wedge \cdots \wedge x)}_{m_{j}} \otimes \underbrace{(x \wedge x \wedge x \wedge \cdots \wedge x)}_{p_{j}},
\end{aligned}
$$

we have $\left|b_{j}^{i}\right|=2\left(m_{j}+p_{j}\right)$. 
Thus $|y|=2 \alpha+q, \quad \alpha=\sum_{i=1}^{r} \sum_{j}^{l_{i}}\left(m_{j}+p_{j}\right) ; \quad q=r-\sum_{i=1}^{r} l_{i}$ and $\left|\tilde{\kappa}_{\Lambda(x)}(y)\right|=$ $2 \alpha+q$, and finally:

$$
\tilde{\kappa}_{\Lambda(x)}(y)\left(e_{k} \tau\right)= \begin{cases}x^{\alpha-\beta} & \text { if } 2 \beta=k-q \\ 0 & \text { if not. }\end{cases}
$$

In particular, for any $y \in\{<[x \otimes 1]>,<[1 \otimes x]>\}$, we obtain:

$$
\tilde{\kappa}_{\Lambda(x)}(y)\left(e_{k} \tau\right)= \begin{cases}x & \text { if } k=0 \\ 0 & \text { if } k>0\end{cases}
$$

and for $y=<[x \otimes x]>$

$$
\tilde{\kappa}_{\Lambda(x)}(y)\left(e_{k} \tau\right)= \begin{cases}x^{2} & \text { if } k=0 \\ x & \text { if } k=2 \\ 0 & \text { if } k \notin\{0,2\}\end{cases}
$$

Then $\left((\Lambda(x), 0), \mu_{\Lambda(x)}, \tilde{\kappa}_{\Lambda(x)}\right)$ has a 1-connected $\pi$-shc minimal model $\left(\left(T V, d_{V}\right), \mu_{V}\right.$, $\left.\widetilde{\kappa}_{V}\right)$ where $V=x \mathbb{F}_{2}, \widetilde{\kappa}_{V}: T \widehat{V} \longrightarrow \operatorname{Hom}(W, T V)$ is the map such that $\widehat{V}=V[2]:=$ $x^{\prime} \mathbb{F}_{2} \oplus x^{\prime \prime} \mathbb{F}_{2} \oplus x^{\prime} \mathbb{F}_{2} \# x^{\prime \prime} \mathbb{F}_{2}$ and

$$
\begin{gathered}
\tilde{\kappa}_{\hat{V}}\left(x^{\prime}\right)\left(e_{i}\right)=\tilde{\kappa}_{\hat{V}}\left(x^{\prime}\right)\left(e_{i} \tau\right)=\tilde{\kappa}_{\hat{V}}\left(x^{\prime \prime}\right)\left(e_{i}\right)=\tilde{\kappa}_{\hat{V}}\left(x^{\prime \prime}\right)\left(e_{i} \tau\right)= \begin{cases}x & \text { if } i=0 \\
0 & \text { if } i>0\end{cases} \\
\tilde{\kappa}_{\hat{V}}\left(x^{\prime} \sharp x^{\prime \prime}\right)\left(e_{i}\right)=\tilde{\kappa}_{\hat{V}}\left(x^{\prime} \sharp x^{\prime \prime}\right)\left(e_{i} \tau\right)= \begin{cases}x & \text { if } i=1 \\
0 & \text { if not. }\end{cases}
\end{gathered}
$$

Let $T \hat{V} \stackrel{q_{\hat{V}}}{\longrightarrow}(T(x))^{\otimes 2}$ be the surjective quasi-isomorphism of differential graded algebras defined on the generic elements as follows: $q_{\hat{V}}\left(x^{\prime}\right)=x \otimes 1 ; q_{\hat{V}}\left(x^{\prime \prime}\right)=1 \otimes x$; and $q_{\hat{V}}\left(x^{\prime} \sharp x^{\prime \prime}\right)=0$, and inducing the chain complex quasi-isomorphism $C^{-} T \hat{V}^{C^{-} q_{\hat{V}}}$ $C^{-}(T(x))^{\otimes 2}$. We have the following diagram

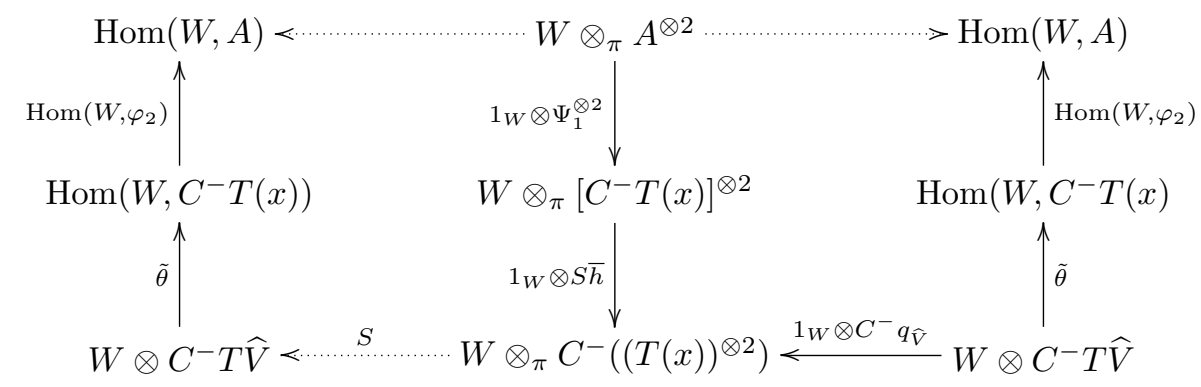


where

$$
\begin{aligned}
A & =\frac{\mathbb{F}_{p}[u] \otimes\left[\mathbb{F}_{p} \oplus_{i=1}^{p-1} \mathbb{F}_{p}<z_{r}>\right] \otimes \Lambda(y)}{<u \otimes z_{r}, r \neq p-1>} \\
& \cong H_{S^{1}}^{*}\left(L \mathbb{C P}(\infty), \mathbb{F}_{p}\right) \\
& \cong H C_{*}^{-}\left(N^{*}\left(\mathbb{C P}(\infty), \mathbb{F}_{p}\right)\right) \\
& \cong H C_{*}^{-}(\Lambda(x)) \\
& \cong H C_{*}^{-} T(x),
\end{aligned}
$$

with $|u|=2,|y|=2 p, z_{r}=2 r+1$ and $\mathbb{F}_{p}<z_{r}>$ the graded vector space generated by $z_{r}$ (see [19, Theorem 2]). $\overline{S h}$ is the homomorphism of chain complexes defined in Section 1, $S$ a linear section of $1_{W} \otimes C^{-} q_{\hat{V}}$; the structural map $\tilde{\theta}$ is defined by $\tilde{\theta}=\phi_{\Lambda(x)} \circ C^{-} \tilde{\kappa}_{\hat{V}}$ and $\varphi_{2}$ is defined as follows: The linear differential map

$$
\mathbb{K}[u] \otimes\left[\mathbb{K} \oplus\left(\oplus_{i=0}^{p-1} \mathbb{K}<z_{r}>\right)\right] \otimes \Lambda(x) \stackrel{\bar{\varphi}}{\longrightarrow} \mathbb{K}[u] \otimes \Lambda(x) \otimes \Lambda(s x)
$$

factors through

$$
A \longrightarrow \mathbb{K}[u] \otimes \Lambda(x) \otimes \Lambda(s x)
$$

and

$$
\mathbb{K}[u] \otimes \Lambda(x) \otimes \Lambda(s x) \stackrel{\psi}{\longrightarrow} A
$$

where $\varphi$ is a differential graded algebras quasi-isomorphism defined in $[\mathbf{1 9}, 4.1]$ or $[\mathbf{1 8}, 4.6]$.

Consider the differential graded algebras quasi-isomorphisms

$$
\mathbb{K}[u] \otimes \Lambda(x) \otimes \Gamma(s x) \underset{\overleftarrow{\theta}}{\stackrel{\tau}{\rightleftarrows}} \mathbb{K}[u] \otimes \mathfrak{C}(\Lambda(x))=\mathcal{C}^{-} \Lambda(x)
$$

(see $[\mathbf{1 8}, 4]$ or $[\mathbf{1 9}, 3.2])$.

From this we define the chain complex homomorphisms

$$
A \underset{\overline{\varphi_{2}}}{\stackrel{\varphi_{1}}{\rightleftarrows}} C_{*}^{-}(\Lambda(x))
$$

such that $\varphi_{1}=\varphi \circ \tau$ and $\varphi_{2}=\psi \circ \bar{\theta}$.

More precisely we have:

(i)

$$
\begin{gathered}
\varphi_{1}(u)=\tau \circ \bar{\varphi}(u)=\tau(u \otimes 1)=u \otimes 1[] \\
\varphi_{1}(y)=\tau \circ \bar{\varphi}(1 \otimes y)=\tau\left(1 \otimes x^{p}\right)=1 \otimes x^{p}[] \\
\forall r \in\{0, \ldots, p-1\}, \quad \varphi_{1}\left(z_{r}\right)=\tau \circ \bar{\varphi}\left(z_{r}\right)=\tau\left(x^{r} \otimes s x\right)=1 \otimes x^{r}[x] .
\end{gathered}
$$

(ii)

$$
\varphi_{2}\left(u^{l} \otimes x^{n}\left[x^{k}\right]\right)=\psi \circ \bar{\theta}\left(u^{l} \otimes x^{n}\left[x^{k}\right]\right)=k \psi\left(u^{l} \otimes x^{k+n-1} \otimes s x\right)
$$


for $q>0$

$$
\begin{gathered}
\varphi_{2}\left(u^{l} \otimes x^{n}\left[x^{k_{1}}\left|x^{k_{2}}\right| \cdots\left|x^{k_{q-1}}\right| x^{k_{q}}\right]\right)=0 . \\
\varphi_{2}\left(u^{l} \otimes x^{n}[]\right)=\psi \circ \bar{\theta}\left(u^{l} \otimes x^{n}[]\right)= \begin{cases}u^{l} \otimes y^{k} & \text { if } n=k p \\
0 & \text { if } n \neq k p .\end{cases}
\end{gathered}
$$

6.1.1. Finally, observe that $S$, the linear section of $1_{W} \otimes C^{-} q_{\hat{V}}$, is only defined in low degrees as follows:

1. $S\left(e_{i} \otimes u^{l} \otimes 1[]\right)=e_{i} \otimes u^{l} \otimes 1[]$

2. $S\left(e_{i} \otimes u^{l} \otimes\left(x^{k} \otimes 1\right)[]\right)=e_{i} \otimes u^{l} \otimes x^{\prime k}[]$

3. $S\left(e_{i} \otimes u^{l} \otimes\left(1 \otimes x^{k}\right)[]\right)=e_{i} \otimes u^{l} \otimes x^{\prime \prime k}[]$

4. $S\left(e_{i} \otimes u^{l} \otimes\left(x^{k} \otimes x^{k^{\prime}}\right)[]\right)=e_{i} \otimes u^{l} \otimes\left(x^{\prime k} x^{\prime k^{\prime}}\right)[]$

5. $S\left(e_{i} \otimes u^{l} \otimes\left(x^{k} \otimes 1\right)\left[x^{k^{\prime}} \otimes 1\right]\right)=e_{i} \otimes u^{l} \otimes x^{\prime k}\left[x^{\prime k^{\prime}}\right]$

6. $S\left(e_{i} \otimes u^{l} \otimes\left(1 \otimes x^{k}\right)\left[1 \otimes x^{k^{\prime}}\right]\right)=e_{i} \otimes u^{l} \otimes x^{\prime \prime k}\left[x^{\prime \prime k^{\prime}}\right]$

7. $S\left(e_{i} \otimes u^{l} \otimes 1\left[x^{k} \otimes 1\right]\right)=e_{i} \otimes u^{l} \otimes 1\left[x^{\prime k}\right]$

8. $S\left(e_{i} \otimes u^{l} \otimes 1\left[1 \otimes x^{k}\right]\right)=e_{i} \otimes u^{l} \otimes 1\left[x^{\prime \prime k}\right]$

9. $S\left(e_{i} \otimes u^{l} \otimes\left(x^{k} \otimes 1\right)\left[1 \otimes x^{k^{\prime}}\right]\right)=e_{i} \otimes u^{l} \otimes x^{\prime k}\left[x^{\prime \prime k^{\prime}}\right]$

10. $S\left(e_{i} \otimes u^{l} \otimes\left(1 \otimes x^{k}\right)\left[x^{k^{\prime}} \otimes 1\right]\right)=e_{i} \otimes u^{l} \otimes x^{\prime \prime k}\left[x^{\prime k^{\prime}}\right]$

11. $S\left(e_{i} \otimes u^{l} \otimes 1\left[1 \otimes x^{k} \mid 1 \otimes x^{k^{\prime}}\right]\right)=e_{i} \otimes u^{l} \otimes 1\left[x^{\prime \prime k} \mid x^{\prime k^{\prime}}\right]$

12. $S\left(e_{i} \otimes u^{l} \otimes 1\left[x^{k} \otimes 1 \mid x^{k^{\prime}} \otimes 1\right]\right)=e_{i} \otimes u^{l} \otimes 1\left[x^{\prime k} \mid x^{\prime k^{\prime}}\right]$

13. $S\left(e_{i} \otimes u^{l} \otimes 1\left[1 \otimes x^{k} \mid x^{k^{\prime}} \otimes 1\right]\right)=e_{i} \otimes u^{l} \otimes 1\left[x^{\prime \prime k} \mid x^{\prime k^{\prime}}\right]$

14. $S\left(e_{i} \otimes u^{l} \otimes 1\left[x^{k} \otimes 1 \mid 1 \otimes x^{k^{\prime}}\right]\right)=e_{i} \otimes u^{l} \otimes 1\left[x^{\prime k} \mid x^{\prime \prime k^{\prime}}\right]$

15. $S\left(e_{i} \otimes u^{l} \otimes 1\left[x^{k} \otimes x^{k^{\prime}}\right]\right)=e_{i} \otimes u^{l} \otimes 1\left[x^{\prime k} x^{\prime \prime k^{\prime}}\right](k>1)$

16. $S\left(e_{i} \otimes u^{l} \otimes 1[x \otimes 1 \mid 1 \otimes x]\right)=e_{i} \otimes u^{l} \otimes 1\left[x^{\prime} \mid x^{\prime \prime}\right]+e_{i} \otimes u^{l} \otimes 1\left[x^{\prime} \sharp x^{\prime \prime}\right](k=1)$

17. $S\left(e_{i} \otimes u^{l} \otimes 1\left[x^{k_{1}} \otimes 1\left|x^{k_{2}} \otimes 1\right| 1 \otimes x^{k_{3}} \mid 1 \otimes x^{k_{4}}\right]\right)=e_{i} \otimes u^{l} \otimes 1\left[x^{\prime k_{1}}\left|x^{\prime k_{2}}\right| x^{\prime \prime k_{3}} \mid x^{\prime \prime k_{4}}\right]$

18. $S\left(e_{i} \otimes u^{l} \otimes 1[1 \otimes x|x \otimes x| x \otimes 1]\right)=e_{i} \otimes u^{l} \otimes 1\left[x^{\prime \prime}\left|x^{\prime} x^{\prime \prime}\right| x^{\prime}\right]$

19. $S\left(e_{i} \otimes u^{l} \otimes 1[x \otimes x|x \otimes 1| 1 \otimes x]\right)=e_{i} \otimes u^{l} \otimes 1\left[x^{\prime} \cdot x^{\prime \prime}\left|x^{\prime}\right| x^{\prime \prime}\right]+$ $e_{i} \otimes u^{l} \otimes 1\left[x^{\prime} \cdot x^{\prime \prime} \mid x^{\prime} \sharp x^{\prime \prime}\right]$

20. $S\left(e_{i} \otimes u^{l} \otimes 1[x \otimes x|x \otimes 1| 1 \otimes x]\right)=e_{i} \otimes u^{l} \otimes 1\left[x^{\prime}\left|x^{\prime \prime}\right| x^{\prime} \cdot x^{\prime \prime}\right]+$ $e_{i} \otimes u^{l} \otimes 1\left[x^{\prime} \sharp x^{\prime \prime} \mid x^{\prime} \cdot x^{\prime \prime}\right]$

21. $S\left(e_{i} \otimes u^{l} \otimes 1[x \otimes x|x \otimes 1| 1 \otimes x]\right)=e_{i} \otimes u^{l} \otimes\left(x^{\prime 2} x^{\prime \prime}\right)\left[x^{\prime \prime}\right]+$ $e_{i} \otimes u^{l} \otimes\left(x^{\prime} x^{\prime \prime}\right)\left(x^{\prime} \sharp x^{\prime \prime}\right)[]$

22. $S\left(e_{i} \otimes u^{l} \otimes 1[x \otimes x|x \otimes 1| 1 \otimes x]\right)=e_{i} \otimes u^{l} \otimes\left(x^{\prime} x^{\prime \prime 2}\right)\left[x^{\prime}\right]+$ $e_{i} \otimes u^{l} \otimes\left(x^{\prime} \sharp x^{\prime \prime}\right)\left(x^{\prime} \cdot x^{\prime \prime}\right)[]$.

6.1.2. Now we define algebraic Steenrod operations on $H^{q}(A)$ by the formula $S q^{i}(x)=\operatorname{cl}\left(\tilde{\theta}\left(S \circ\left(i d_{W} \otimes \overline{S h}\right)\left(e_{n-i} \otimes x \otimes x\right)\right)\right), x \in H^{*}(A)$

1. For $x=u$,

(a) $S q^{0}(u)=c l\left[\varphi_{2}(u \otimes 1[])\right]=u=x$.

(b) $S q^{1}(u)=\operatorname{cl}\left[\varphi_{2}\left(g\left(u^{2}\right)\left(e_{1}\right) \otimes \tilde{\kappa}_{\hat{V}}(1)\left(e_{0}\right)[]+g\left(u^{2}\right)\left(e_{0}\right) \otimes \tilde{\kappa}_{\hat{V}}(1)\left(e_{1}\right)[]\right)\right]=0$.

(c) $S q^{2}(u)=\operatorname{cl}\left[\varphi_{2}\left(g\left(u^{2}\right)\left(e_{0}\right) \otimes \tilde{\kappa}_{\hat{V}}(1)\left(e_{0}\right)[]\right)\right]+\operatorname{cl}\left[\varphi_{2}\left(u^{2} \otimes 1[]\right)\right]=x^{2}$. 
(d) for $i>2, S q^{i}(u)=0$.

2. For $x=y$,

(a) $S q^{0}(y)=\operatorname{cl}\left[\varphi_{2}\left(g(1)\left(e_{0}\right) \otimes \tilde{\kappa}_{\hat{V}}\left(x^{\prime 2} x^{\prime \prime 2}\right)\left(e_{4}\right)[]\right)\right]=\operatorname{cl}\left[\varphi_{2}\left(1 \otimes x^{2}[]\right)\right]=$ $1 \otimes 1 \otimes y$.

(b) $S q^{1}(y)=c l\left[\varphi_{2}(I d \otimes I d)\left(g(1) \otimes \tilde{\kappa}_{\hat{V}}\left(x^{\prime 2} x^{\prime \prime 2}\right)\right) \circ \psi_{W}\left(e_{3}\right)\right]=0$.

(c) $S q^{2}(y)=\operatorname{cl}\left[\varphi_{2}(I d \otimes I d)\left(g(1) \otimes \tilde{\kappa}_{\hat{V}}\left(x^{\prime 2} x^{\prime \prime 2}\right)\right) \circ \psi_{W}\left(e_{2}\right)\right]=c l\left[\varphi_{2}\left(1 \otimes x^{3}[]\right)\right]$ $=0$.

(d) $S q^{3}(y)=c l\left[\varphi_{2}(I d \otimes I d)\left(g(1) \otimes \tilde{\kappa}_{\hat{V}}\left(x^{\prime 2} x^{\prime \prime 2}\right)\right) \circ \psi_{W}\left(e_{1}\right)\right]=0$.

(e) $S q^{4}(y)=\operatorname{cl}\left[\varphi_{2}(I d \otimes I d)\left(g(1) \otimes \tilde{\kappa}_{\hat{V}}\left(x^{\prime 2} x^{\prime \prime 2}\right)\right) \circ \psi_{W}\left(e_{0}\right)\right]=\operatorname{cl}\left[\varphi_{2}\left(1 \otimes x^{4}[]\right)\right]$ $=y^{2}$.

(f) for $i>4=2 p, S q^{i}(y)=0$.

3. For $x=z_{r} \in H^{2 r+1}(A)$ with $0 \leqslant r \leqslant p-1$, since $p=2$ then $r \in\{0,1\}$.

(a) $x=z_{0}$

i. $\quad S q^{0}\left(z_{0}\right)=\operatorname{cl}\left[\varphi_{2}\left(\left(I d \otimes I d \otimes s^{\otimes 2}\right)\left(g(1) \otimes \tilde{\kappa}_{\hat{V}}(1) \otimes \tilde{\kappa}_{\hat{V}}\left(x^{\prime}\right) \otimes \tilde{\kappa}_{\hat{V}}(1) \otimes\right.\right.\right.$ $\left.\left.\tilde{\kappa}_{\hat{V}}\left(x^{\prime \prime}\right) \otimes \tilde{\kappa}_{\hat{V}}\left(x^{\prime}\right)\right)\right) \psi_{W}^{(3)}\left(e_{1}\right)+$ $\left.\left.I d \otimes I d \otimes s^{\otimes 2}\right)\left(g(1) \otimes \tilde{\kappa}_{\hat{V}}(1) \otimes \tilde{\kappa}_{\hat{V}}\left(x^{\prime} \sharp x^{\prime \prime}\right)\right) \psi_{W}^{(2)}\left(e_{1}\right)\right]$ $=S q^{0}\left(z_{0}\right) c l\left[\varphi_{2}(1 \otimes 1[x])\right]$ $=z_{0}$.

ii. $\quad S q^{1}\left(z_{0}\right)=\operatorname{cl}\left[\varphi_{2}\left(\left(I d \otimes I d \otimes s^{\otimes 2}\right)\left(g(1) \otimes \tilde{\kappa}_{\hat{V}}(1) \otimes \tilde{\kappa}_{\hat{V}}\left(x^{\prime}\right) \otimes \tilde{\kappa}_{\hat{V}}\left(x^{\prime \prime}\right)+\right.\right.\right.$ $\begin{aligned} & \left.\left.g(1) \otimes \tilde{\kappa}_{\hat{V}}(1) \otimes \tilde{\kappa}_{\hat{V}}\left(x^{\prime \prime}\right) \otimes \tilde{\kappa}_{\hat{V}}\left(x^{\prime}\right)\right)\right) \psi_{W}^{(3)}\left(e_{0}\right)+ \\ & \left.\left(I d \otimes I d \otimes s^{\otimes 2}\right)\left(g(1) \otimes \tilde{\kappa}_{\hat{V}}(1) \otimes \tilde{\kappa}_{\hat{V}}\left(x^{\prime} \sharp x^{\prime \prime}\right)\right) \psi_{W}^{(2)}\left(e_{0}\right)\right] \\ = & c l\left[\varphi_{2}(2(1 \otimes 1[x \mid x]))\right]=z_{0}^{2}=0 .\end{aligned}$

iii. for $i>1, S q^{i}\left(z_{0}\right)=0$.

(b) $x=z_{1}$.

i. $S q^{0}\left(z_{1}\right)=z_{1}$.

ii. $S q^{1}\left(z_{1}\right)=\operatorname{cl}\left[\varphi_{2}\left(\left(I d \otimes I d \otimes s^{\otimes 2}\right)\left(g(1) \otimes \tilde{\kappa}_{\hat{V}}\left(x^{\prime} x^{\prime \prime}\right) \otimes \tilde{\kappa}_{\hat{V}}\left(x^{\prime}\right) \otimes \tilde{\kappa}_{\hat{V}}\left(x^{\prime \prime}\right)\right.\right.\right.$ $\left.\left.+g(1) \otimes \tilde{\kappa}_{\hat{V}}\left(x^{\prime} x^{\prime \prime}\right) \otimes \tilde{\kappa}_{\hat{V}}\left(x^{\prime \prime}\right) \otimes \tilde{\kappa}_{\hat{V}}\left(x^{\prime}\right)\right)\right) \psi_{W}^{(3)}\left(e_{2}\right)+$ $\left.\left(I d \otimes I d \otimes s^{\otimes 2}\right)\left(g(1) \otimes \tilde{\kappa}_{\hat{V}}\left(x^{\prime} x^{\prime \prime}\right) \otimes \tilde{\kappa}_{\hat{V}}\left(x^{\prime} \sharp x^{\prime \prime}\right)\right) \psi_{W}^{(2)}\left(e_{2}\right)\right]$ $=\operatorname{cl}\left[\varphi_{2}(2(1 \otimes x[x \mid x]))\right]=0$.

iii. $S q^{2}\left(z_{1}\right)=\operatorname{cl}\left[\varphi_{2}\left(\left(I d \otimes I d \otimes s^{\otimes 2}\right)\left(g(1) \otimes \tilde{\kappa}_{\hat{V}}\left(x^{\prime} x^{\prime \prime}\right) \otimes \tilde{\kappa}_{\hat{V}}\left(x^{\prime}\right) \otimes \tilde{\kappa}_{\hat{V}}\left(x^{\prime \prime}\right)\right.\right.\right.$

$$
\begin{aligned}
& \left.\left.+g(1) \otimes \tilde{\kappa}_{\hat{V}}\left(x^{\prime} x^{\prime \prime}\right) \otimes \tilde{\kappa}_{\hat{V}}\left(x^{\prime \prime}\right) \otimes \tilde{\kappa}_{\hat{V}}\left(x^{\prime}\right)\right)\right) \psi_{W}^{(3)}\left(e_{1}\right)+ \\
& \left.\left(I d \otimes I d \otimes s^{\otimes 2}\right)\left(g(1) \otimes \tilde{\kappa}_{\hat{V}}\left(x^{\prime} x^{\prime \prime}\right) \otimes \tilde{\kappa}_{\hat{V}}\left(x^{\prime} \sharp x^{\prime \prime}\right)\right) \psi_{W}^{(2)}\left(e_{1}\right)\right] \\
= & c l\left[\varphi_{2}\left(1 \otimes x_{2}[x \mid x]\right)\right] \\
= & y \otimes z_{0} .
\end{aligned}
$$

iv. $S q^{3}\left(z_{1}\right)=\operatorname{cl}\left[\varphi_{2}\left(2\left(1 \otimes x^{2}[x \mid x]\right)\right)\right]=0$.

v. for $i>3, S q^{i}\left(z_{1}\right)=0$. 


\subsection{Odd sphere $S^{2 q+1}$}

As in the previous example, let $X=S^{2 q+1}, H^{*}(X ; \mathbb{K})=\Lambda(x)=\mathbb{F}_{2}[x]$ with $|x|=$ $2 q+1$. Thus the graded algebras $H C_{*}^{-}\left(N^{*} S^{2 q+1} ; \mathbb{F}_{2}\right)$ and $H C_{*}^{-}(\Lambda(x))$ are isomorphic. It also follows that the algebraic Steenrod operations on $H C_{*}^{-}\left(N^{*} S^{2 q+1} ; \mathbb{F}_{2}\right)$ are computed by those on $H C_{*}^{-}(\Lambda(x))$.

Since $\left((\Lambda(x), 0), \mu_{\Lambda(x)}, \tilde{\kappa}_{\Lambda(x)}\right)$ is a 1-connected $\pi$-shc differential graded algebra together with finite generated cohomology groups such that the shc structural map $\mu_{\Lambda(x)}=\Omega B\left(\left(m_{\Lambda(x)}\right)\right)$ and the $\pi$-shc structural map $\Omega B\left(\Lambda(x)^{\otimes 2}\right) \stackrel{\tilde{\kappa}_{\Lambda(x)}}{\longrightarrow} \operatorname{Hom}(W ; \Lambda(x))$ are defined by the following diagram

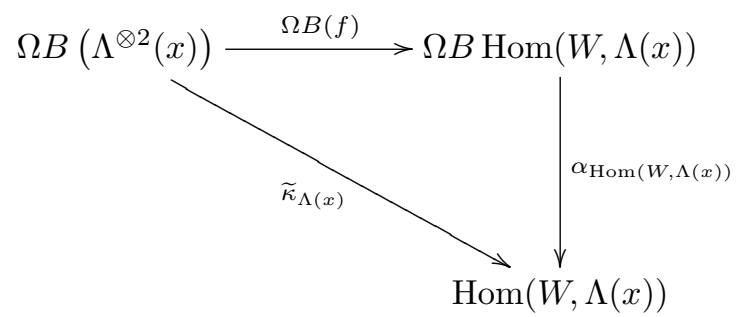

which induces the diagram

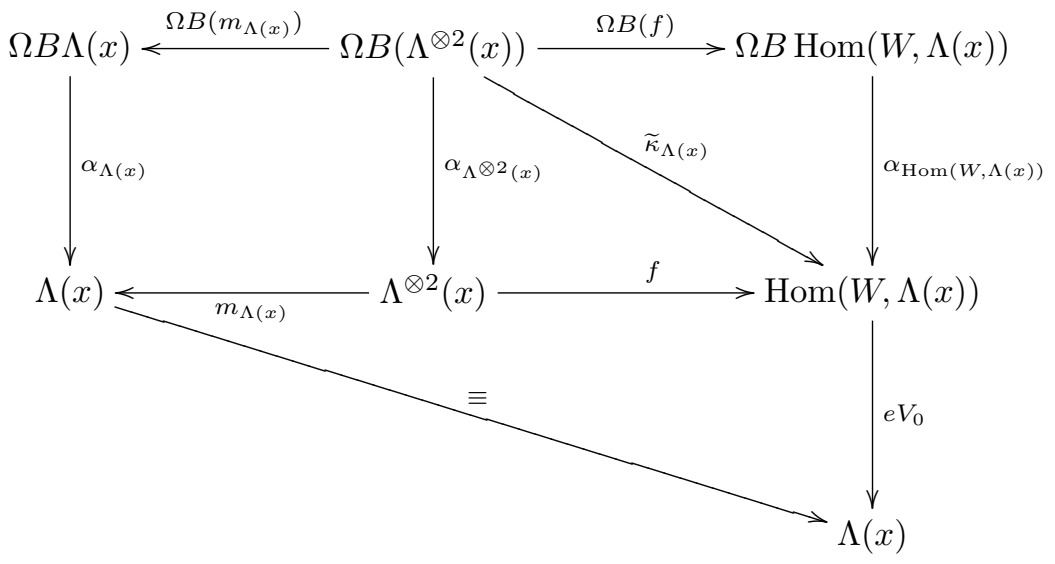

where $f$ is defined by

$$
\begin{array}{r}
f: \Lambda^{\otimes 2}(x) \longrightarrow \operatorname{Hom}(W, \Lambda(x)) \\
x \otimes x \longmapsto f(x \otimes x)=f_{x \otimes x} \\
1 \otimes x \longmapsto f(1 \otimes x)=f_{1 \otimes x} \\
x \otimes 1 \longmapsto f(x \otimes 1)=f_{x \otimes 1}
\end{array}
$$


such that

$$
\begin{gathered}
f_{x \otimes x}\left(e_{i}\right)=f_{x \otimes x}\left(\tau e_{i}\right)= \begin{cases}x & \text { if } i=2 q+1 \\
0 & \text { if } i \neq 2 q+1\end{cases} \\
f_{1 \otimes x}\left(e_{i}\right)=f_{1 \otimes x}\left(\tau e_{i}\right)= \begin{cases}x & \text { if } i=0 \\
0 & \text { if } i \neq 0\end{cases} \\
f_{x \otimes 1}\left(e_{i}\right)=f_{x \otimes 1}\left(\tau e_{i}\right)= \begin{cases}x & \text { if } i=0 \\
0 & \text { if } i \neq 0\end{cases} \\
f_{1}\left(e_{i}\right)=f_{1}\left(\tau e_{i}\right)= \begin{cases}1 & \text { if } i=0 \\
0 & \text { if } i \neq 0\end{cases}
\end{gathered}
$$

where $f_{1}$ is the unit of the differential graded algebra $\operatorname{Hom}(W, \Lambda(x))$.

Then $\left((\Lambda(x), 0), \mu_{\Lambda(x)}, \tilde{\kappa}_{\Lambda(x)}\right)$ has a 1-connected $\pi$-shc minimal model $\left(\left(T V, d_{V}\right)\right.$, $\left.\mu_{V}, \widetilde{\kappa}_{V}\right)$ where $V=x \mathbb{F}_{2}, \widetilde{\kappa}_{V}: T \widehat{V} \longrightarrow \operatorname{Hom}(W, T V)$ a map such that $\widehat{V}=V[2]:=$ $V^{\prime} \oplus V^{\prime \prime} \oplus V^{\prime} \# V^{\prime \prime} \quad$ with $V^{\prime}:=s^{-1}(s V \otimes \mathbb{K})=s^{-1}\left(H^{+} B \Lambda(x) \otimes \mathbb{K}\right) ; V^{\prime \prime}=s^{-1}(\mathbb{K} \otimes$ $s V)=s^{-1}\left(\mathbb{K} \otimes H^{+} B \Lambda(x)\right) ; V^{\prime} \# V^{\prime \prime}=s^{-1}(s V \otimes s V)=s^{-1}\left(H^{+} B \Lambda(x) \otimes H^{+} B \Lambda(x)\right)$, where $a_{k_{1}}^{\prime}, a_{k_{2}}^{\prime \prime}, a_{k_{1}}^{\prime} \# a_{k_{2}}^{\prime \prime}$ are the respective generators of $V^{\prime}, V^{\prime \prime}, V^{\prime} \# V^{\prime \prime}$;

$$
a_{k_{1}}^{\prime}:=s^{-1}\left(s a_{k_{1}} \otimes 1\right) ; \quad a_{k_{2}}^{\prime \prime}:=s^{-1}\left(1 \otimes s a_{k_{2}}\right) ; \quad a_{k_{1}}^{\prime} \# a_{k_{2}}^{\prime \prime}:=s^{-1}\left(s a_{k_{1}} \otimes s a_{k_{2}}\right) ;
$$

with $a_{k_{1}}:=\underbrace{[x|\cdots| x]}_{k_{1} \text { times }} ; a_{k_{2}}:=\underbrace{[x|\cdots| x]}_{k_{2} \text { times }} ;\left|a_{k_{1}}^{\prime}\right|=2 q k_{1}+1 ;\left|a_{k_{2}}^{\prime \prime}\right|=2 q k_{2}+1 ;\left|a_{k_{1}}^{\prime} \# a_{k_{2}}^{\prime \prime}\right|=$ $2 q\left(k_{1}+k_{2}\right)+1$. And

$$
\widetilde{\kappa}_{\widehat{V}}\left(a_{k_{1}}^{\prime}\right)= \begin{cases}f_{1} & \text { if } k_{1}=0 \\ f_{1 \otimes x} & \text { if } k_{1}=1 \\ 0 & \text { if not. }\end{cases}
$$

Since $\left|a_{k_{1}}^{\prime}\right|=2 q+1 ; \widetilde{\kappa}_{\widehat{V}}\left(a_{k_{1}}^{\prime}\right)\left(\tau^{j} e_{i}\right)=\left\{\begin{array}{ll}x & \text { if } i=0 \\ 0 & \text { if } i \neq 0 ;\end{array} \quad j \geqslant 0\right.$,

$$
\widetilde{\kappa}_{\widehat{V}}\left(a_{k_{1}}^{\prime}\right)= \begin{cases}f_{1} & \text { if } k_{2}=0 \\ f_{1 \otimes x} & \text { if } k_{2}=1 \\ 0 & \text { if not }\end{cases}
$$

since $\left|a_{k_{2}}^{\prime \prime}\right|=2 q+1, \widetilde{\kappa}_{\widehat{V}}\left(a_{1}^{\prime \prime}\right)\left(\tau^{j} e_{i}\right)= \begin{cases}x & \text { if } i=0 \\ 0 & \text { if } i \neq 0\end{cases}$

$$
\widetilde{\kappa}_{\widehat{V}}\left(a_{k_{1}}^{\prime} \# a_{k_{2}}^{\prime \prime}\right)\left(\tau^{j} e_{i}\right)= \begin{cases}x & \text { if } i=2 \quad k_{1}=k_{2}=1 \\ 0 & \text { if not. }\end{cases}
$$

Let $T \hat{V} \stackrel{q_{\hat{V}}}{\longrightarrow}(T(x))^{\otimes 2}$ be the surjective quasi-isomorphism of differential graded algebras defined on the generic elements as follows:

$$
q_{\widehat{V}}\left(a_{k_{1}}^{\prime}\right)=s^{-1} \underbrace{[x|\cdots| x]}_{k_{1} \text { times }} \otimes 1 ; \quad q_{\widehat{V}}\left(a_{k_{2}}^{\prime \prime}\right)=1 \otimes s^{-1} \underbrace{[x|\cdots| x]}_{k_{2} \text { times }} ; \quad q_{\widehat{V}}\left(a_{k}^{\prime} \# a_{k}^{\prime \prime}\right)=0 .
$$


and inducing the chain complex quasi-isomorphism $C^{-} T \hat{V}^{C^{-} q_{\hat{v}}} C^{-}(T(x))^{\otimes 2}$. We have the following diagram

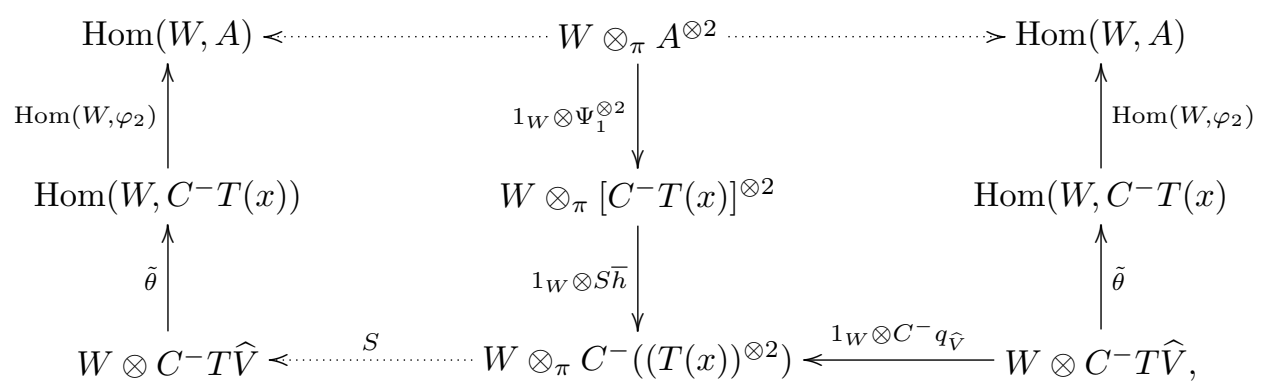

where

$$
\begin{aligned}
A=\frac{\mathbb{K}[u] \otimes \Lambda(y) \otimes \Gamma(s x)}{\left\langle u \otimes \gamma^{n}(s x), \gamma^{n}(s x) \otimes y\right\rangle, n \neq k p} & \cong H^{-*}(\mathbb{K}[u] \otimes \Lambda(x) \otimes \Gamma(s x)) \\
& \cong H_{S^{1}}^{-*}(L X, \mathbb{K})
\end{aligned}
$$

with $|u|=2 ;\|x\|=2 q+1 ;|y|=2 q p+1$ [19], see [19, Theorem 2]. $S$ is the section of $1_{W} \otimes C^{-} q_{\widehat{V}} ; \widetilde{\theta}=\phi_{\Lambda(x)} \circ C^{-} \widetilde{\kappa}_{\widehat{V}}$ and $\varphi_{2}$ is the map defined as follows: Consider the differential algebra homomorphisms

$$
\mathbb{K}[u] \otimes \Lambda(x) \otimes \Gamma(s x) \underset{\bar{\theta}}{\stackrel{\tau}{\rightleftarrows}} \mathbb{K}[u] \otimes \mathcal{C}(\Lambda(x))=\mathcal{C}^{-} \Lambda(x) .
$$

respectively defined by:

(i) $\tau(1 \otimes x \otimes 1)=1 \otimes x[] ; \quad \tau\left(u^{l} \otimes 1 \otimes 1\right)=u^{l} \otimes 1[]$

$$
\tau\left(1 \otimes 1 \otimes \gamma^{k}(s x)\right)=1 \otimes \underbrace{[x|\cdots| x]}_{(k) \text { times }} ; \quad \tau\left(u^{l} \otimes x \otimes \gamma^{k}(s x)\right)=u^{l} \otimes x \underbrace{[x|\cdots| x]}_{k \text { times }}
$$

(ii) $\bar{\theta}\left(u^{l} \otimes 1[]\right)=u^{l} \otimes 1 \otimes 1 ; \quad \bar{\theta}(1 \otimes 1 \underbrace{[x|\cdots| x]}_{(k) \text { times }})=1 \otimes 1 \otimes \gamma^{k}(s x)$

$$
\bar{\theta}(1 \otimes x[])=1 \otimes x \otimes 1 ; \quad \bar{\theta}(u^{l} \otimes x \underbrace{[x|\cdots| x]}_{(k) \text { times }})=u^{l} \otimes x \otimes \gamma^{k}(s x) .
$$

$\tau$ and $\bar{\theta}$ are homotopic equivalence algebras inverse to each other and $H(\bar{\theta})=H(\tau)^{-1}$. From this differential graded algebra quasi-isomorphism we deduce the following chain complex homomorphisms:

$$
C^{-}(\Lambda(x)) \underset{\varphi_{2}}{\stackrel{\varphi_{1}}{\leftrightarrows}} A
$$

$\varphi_{1}=\tau \circ \phi$ et $\varphi_{2}=\Psi \circ \bar{\theta}$. More precisely, we have:

1.

$$
\begin{gathered}
\varphi_{1}(u)=\tau \circ \varphi(u)=u \otimes 1[] ; \\
\varphi_{1}(y)=\tau \circ \varphi(y)=\tau\left(x \otimes \gamma^{p-1}(s x)\right)=1 \otimes x \underbrace{[x|\cdots| x]}_{(p-1) \text { times }} ; \\
\varphi_{1}(s x)=\tau \circ \phi\left(\gamma^{1}(s x)\right)=\tau\left(\gamma^{1}(s x)\right)=\tau(s x)=1 \otimes 1[x]
\end{gathered}
$$


2.

$$
\begin{aligned}
& \varphi_{2}\left(u^{l} \otimes 1[]\right)=\Psi \circ \bar{\theta}\left(u^{l} \otimes 1 \otimes 1\right)=u^{l} \otimes 1 \otimes 1 \\
& \varphi_{2}(1 \otimes x[])=\Psi \circ \bar{\theta}(1 \otimes x[])=\Psi(1 \otimes x \otimes 1)=0 \\
& \varphi_{2}(1 \otimes 1 \underbrace{[x|\cdots| x]}_{\text {ntimes }})=\Psi \circ \bar{\theta}(1 \otimes 1 \underbrace{[x|\cdots| x]}_{\text {ntimes }}) \\
& =\Psi\left(1 \otimes 1 \otimes \gamma^{n}(s x)\right) \\
& =1 \otimes 1 \otimes \gamma^{n}(s x) \\
& \varphi_{2}(u^{l} \otimes x \underbrace{[x|\cdots| x]}_{\text {ntimes }}) \\
& =\Psi\left(u^{l} \otimes x \otimes \gamma^{n}(S x)\right)= \begin{cases}\left(C_{n}^{p-1}\right)^{-1} u^{l} \otimes y \otimes \gamma^{n-p+1}(s x) & \text { if } n+1=k p \\
0 & \text { if } n+1 \neq k p\end{cases}
\end{aligned}
$$

(see $[\mathbf{1 9}])$.

6.2.1. Finally, we observe that $S$, the linear section of $1_{W} \otimes C^{-} q_{\hat{V}}$, is only defined in low degrees as follows:

1. $S\left(e_{i} \otimes u^{l} \otimes 1[]\right)=e_{i} \otimes u^{l} \otimes 1[]$

2. $S\left(e_{i} \otimes u^{l} \otimes 1[x \otimes 1 \mid 1 \otimes x]\right)=e_{i} \otimes u^{l} \otimes 1\left[a_{1}^{\prime} \mid a_{1}^{\prime \prime}\right]+e_{i} \otimes u^{l} \otimes 1\left[a_{1}^{\prime} \# a_{1}^{\prime \prime}\right]$

3. $S\left(e_{i} \otimes u^{l} \otimes 1[x \otimes 1]\right)=e_{i} \otimes u^{l} \otimes 1\left[a_{1}^{\prime}\right]$

4. $S\left(e_{i} \otimes u^{l} \otimes 1[1 \otimes x]\right)=e_{i} \otimes u^{l} \otimes 1\left[a_{1}^{\prime \prime}\right]$

5. $S\left(e_{i} \otimes u^{l} \otimes 1[x \otimes x]\right)=e_{i} \otimes u^{l} \otimes 1\left[a_{1}^{\prime} \# a_{1}^{\prime \prime}\right]+e_{i} \otimes u^{l} \otimes 1\left[a_{1}^{\prime} \cdot a_{1}^{\prime \prime}\right]$

6. $S\left(e_{i} \otimes u^{l} \otimes x \otimes 1[1 \otimes x]\right)=e_{i} \otimes u^{l} \otimes a_{1}^{\prime}\left[a_{1}^{\prime \prime}\right]$

7. $S\left(e_{i} \otimes u^{l} \otimes 1 \otimes x[x \otimes 1]\right)=e_{i} \otimes u^{l} \otimes a_{1}^{\prime \prime}\left[a_{1}^{\prime}\right]$

8. $S\left(e_{i} \otimes u^{l} \otimes 1[1 \otimes x \mid x \otimes 1]\right)=e_{i} \otimes u^{l} \otimes 1\left[a_{1}^{\prime \prime} \mid a_{1}^{\prime}\right]$

9. $S\left(e_{i} \otimes u^{l} \otimes x \otimes x[x \otimes x]\right)=e_{i} \otimes u^{l} \otimes a_{1}^{\prime} \cdot a_{1}^{\prime \prime}\left[a_{1}^{\prime} \cdot a_{1}^{\prime \prime}\right]$

10. $S\left(e_{i} \otimes u^{l} \otimes x^{2} \otimes x[1 \otimes x]\right)=e_{i} \otimes u^{l} \otimes a_{1}^{\prime 2} \cdot a_{1}^{\prime \prime}\left[a_{1}^{\prime \prime}\right]$

11. $S\left(e_{i} \otimes u^{l} \otimes x \otimes x^{2}[x \otimes 1]\right)=e_{i} \otimes u^{l} \otimes a_{1}^{\prime} \cdot a_{1}^{\prime \prime 2}\left[a_{1}^{\prime}\right]$

12. $S\left(e_{i} \otimes u^{l} \otimes 1[x \otimes 1|1 \otimes x| x \otimes 1 \mid 1 \otimes x]\right)=e_{i} \otimes u^{l} \otimes 1\left[a_{1}^{\prime}\left|a_{1}^{\prime \prime}\right| a_{1}^{\prime} \mid a_{1}^{\prime \prime}\right]$

13. $S\left(e_{i} \otimes u^{l} \otimes 1[1 \otimes x|x \otimes 1| 1 \otimes x \mid x \otimes 1]\right)=e_{i} \otimes u^{l} \otimes 1\left[a_{1}^{\prime \prime}\left|a_{1}^{\prime}\right| a_{1}^{\prime \prime} \mid a_{1}^{\prime}\right]$

14. $S\left(e_{i} \otimes u^{l} \otimes 1[x \otimes x|x \otimes 1| 1 \otimes x]\right)=e_{i} \otimes u^{l} \otimes 1\left[a_{1}^{\prime} \cdot a_{1}^{\prime \prime}\left|a_{1}^{\prime}\right| a_{1}^{\prime \prime}\right]$

15. $S\left(e_{i} \otimes u^{l} \otimes 1[1 \otimes x|x \otimes x| x \otimes 1]\right)=e_{i} \otimes u^{l} \otimes 1\left[a_{1}^{\prime \prime}\left|a_{1}^{\prime} \cdot a_{1}^{\prime \prime}\right| a_{1}^{\prime}\right]$

16. $S\left(e_{i} \otimes u^{l} \otimes 1[x \otimes 1|1 \otimes x| x \otimes x]\right)=e_{i} \otimes u^{l} \otimes 1\left[a_{1}^{\prime}\left|a_{1}^{\prime \prime}\right| a_{1}^{\prime} \cdot a_{1}^{\prime \prime}\right]$

17. $S\left(e_{i} \otimes u^{l} \otimes x \otimes x[x \otimes 1 \mid 1 \otimes x]\right)=e_{i} \otimes u^{l} \otimes a_{1}^{\prime} \cdot a_{1}^{\prime \prime}\left[a_{1}^{\prime} \mid a_{1}^{\prime \prime}\right]$

18. $S\left(e_{i} \otimes 1 \otimes 1 \otimes x[x \otimes 1|1 \otimes x| x \otimes 1]\right)=e_{i} \otimes 1 \otimes a_{1}^{\prime \prime}\left[a_{1}^{\prime}\left|a_{1}^{\prime \prime}\right| a_{1}^{\prime}\right]$

19. $S\left(e_{i} \otimes 1 \otimes x \otimes 1[1 \otimes x|x \otimes 1| 1 \otimes x]\right)=e_{i} \otimes 1 \otimes a_{1}^{\prime}\left[a_{1}^{\prime \prime}\left|a_{1}^{\prime}\right| a_{1}^{\prime \prime}\right]$. 
6.2.2. Now we define algebraic Steenrod operations on $H^{q}(A)$ by the formula

$$
S q^{i}(x)=\operatorname{cl}\left(\tilde{\theta}\left(S \circ\left(i d_{W} \otimes \overline{S h}\right)\left(e_{n-i} \otimes x \otimes x\right)\right)\right), \quad x \in H^{*}(A) .
$$

If $a \in H^{q}(A), a \in\{u, s x, y\}$

1. $\underline{a=u}$

(a) For $i=0, S q^{0}(u)=c l\left[\varphi_{2}(u \otimes 1[])\right]=u$.

(b) For $i=1, S q^{1}(u)=0$.

(c) For $i=2, S q^{2}(u \otimes 1 \otimes 1)=c l\left[\varphi_{2}\left(u^{2} \otimes 1[]\right)\right]=u^{2}$.

(d) For $i>2, S q^{i}(u \otimes 1 \otimes 1)=0$.

2. $a=s x \in H^{2 q}(A)$

(a) For $i=0, S q^{0}(s x)=c l\left[\varphi_{2}(1 \otimes 1[x])\right]=c l[\Psi \circ \theta(1 \otimes 1[x])]=s x$.

(b) For $i \in\{1, \ldots, 2 q-1\}, S q^{i}(a)=0$.

(c) For $i=2 q$,

$$
\begin{aligned}
S q^{2 q}(s x) & =c l\left[\varphi_{2}(2(1 \otimes 1[x \mid x]))\right] \\
& =c l\left[2\left(1 \otimes 1 \otimes \gamma^{2} s x\right)\right] \\
& =c l\left(C_{2}^{1}\left(1 \otimes 1 \otimes \gamma^{2} s x\right)\right) \\
& =(1 \otimes 1 \otimes \gamma s x)(1 \otimes 1 \otimes \gamma s x) \\
& =0 \\
& =(1 \otimes 1 \otimes s x)^{2} .
\end{aligned}
$$

(d) For $i>2 q, S q^{i}(a)=0$.

3. $a=y \in H^{2 q p+1}(A)$

(a) For $i=0, S q^{0}(y)=c l\left[\varphi_{2}(1 \otimes x \otimes[x])\right]=y$.

(b) For $i=1, S q^{1}(y)=0$.

(c) For $i=2, S q^{2}(a)=0$.

(d) For $i \in\{3, \ldots, 4 q\}, S q^{i}(a)=0$.

(e) For $i=4 q+1, S q^{4 q+1}(a)=0=y^{2}$.

(f) For $i>4 q+1, S q^{i}(a)=0$.

\section{References}

[1] D. Chataur and K. Kuribayashi. An operadic model for a mapping space and its associated spectral sequence, J. Pure Appl. Algebra 210 (2007), 321-342.

[2] D. Chataur and J.-C. Thomas $E_{\infty}$-model for mapping spaces, Topology Appl. 145 (2004), 191-208.

[3] Christine E. Hood and John D.S. Jones Some Algebraic Properties of Cyclic Homology Groups D. Reidel Publishing Kompany, K-Theory 1 (1987), 361-384

[4] A. Dold, Über die Steenrodschen Kohomologieoperationen, Ann. of Math. 73 (1961), 258-294.

[5] M. Elhamdadi and Y. G. Gouda, On the Steenrod operations in cyclic cohomology, Int. J. Math. Math. Sci. 72 (2003), 4539-4545.

[6] Y. Felix, S. Halperin and J. C. Thomas, Adams' cobar equivalence, Trans. Amer. Math. Soc. 329 (1992), 531-549. 
[7] Y. Felix, S. Halperin and J.-C. Thomas, Differential Graded Algebras in Topology, Handbook of algebraic topology, North-Holland, Amsterdam (1995).

[8] E. Getzler and J. Jones, $A_{\infty}$-algebras and cyclic bar complex, Illinois J. Math. 34 no. 2 (1990), 256-283

[9] A. Hamilton and A. Lazarev, Homotopy algebras and non commutative geometry, arXiv:math.QA/0410621.

[10] J.D.S. Jones, Cyclic homology and equivariant homology, Invent. Math. 87 (1987), 403-423.

[11] M. Karoubi, Formes différentielles non commutatives et opération de Steenrod, Topology 34 (1995), 699-715.

[12] A. Liulevicius, The factorisation of cyclic reduced powers by secondary cohomology operations, Mem. Amer. Math. Soc. 42, AMS, Providence, RI, 1962.

[13] J-L. Loday, Cyclic Homology, Grundleren der mathematischen Wissenschaften, 301, Springer, Heidelberg, 1991.

[14] M.A. Mandell, $E_{\infty}$-algebras and p-adic homotopy theory, Topology 40 (2001), $43-94$.

[15] J.P. May, A General Algebraic Approach to Steenrod Operations, Springer Lecture Notes in Math., 168 1970, 153-231.

[16] J. McCleary, A User's Guide of Spectral Sequences Second edition, Cambridge University Press, 2001.

[17] H. J. Munkholm, The Eilenberg-Moore spectral sequence and strongly homotopy multiplicative maps, J. Pure Appl. Algebra 5 (1974), 1-50.

[18] B. Ndombol and M. Elhaouari, On the negative cyclic homology of shc-algebra, Math. Ann. 338 (2007), 338-385.

[19] B. Ndombol and M. El Haouari, The free loop space $S^{1}$-equivariant cohomology algebra of some formal spaces, preprint.

[20] B. Ndombol and J.-C. Thomas, On the cohomology algebra of free loop space, Topology 41 (2002), 85-106.

[21] B. Ndombol and J.-C. Thomas, Steenrod operations in shc-algebras, J. Pure Appl. Algebra 192 (2004), 239-264.

[22] N.E. Steenrod Product of cocycles and extensions of mapping, Ann. Math. 48 (1947), 290-320.

Calvin Tcheka jtcheka@yahoo.fr

Mathematics Department, University of Dschang, PO Box: 67 Dschang, Dschang, 237, Cameroon 